\title{
The Next 700 BFT Protocols
}

\author{
PIERRE-LOUIS AUBLIN, INSA Lyon \\ RACHID GUERRAOUI, EPFL \\ NIKOLA KNEŽEVIĆ, IBM Research - Zurich \\ VIVIEN QUÉMA, Grenoble INP \\ MARKO VUKOLIĆ, Eurécom
}

We present Abstract (ABortable STate mAChine replicaTion), a new abstraction for designing and reconfiguring generalized replicated state machines that are, unlike traditional state machines, allowed to abort executing a client's request if "something goes wrong."

Abstract can be used to considerably simplify the incremental development of efficient Byzantine faulttolerant state machine replication (BFT) protocols that are notorious for being difficult to develop. In short, we treat a BFT protocol as a composition of Abstract instances. Each instance is developed and analyzed independently and optimized for specific system conditions. We illustrate the power of Abstract through several interesting examples.

We first show how Abstract can yield benefits of a state-of-the-art BFT protocol in a less painful and errorprone manner. Namely, we develop AZyzzyva, a new protocol that mimics the celebrated best-case behavior of Zyzzyva using less than 35\% of the Zyzzyva code. To cover worst-case situations, our abstraction enables one to use in AZyzzyva any existing BFT protocol.

We then present Aliph, a new BFT protocol that outperforms previous BFT protocols in terms of both latency (by up to $360 \%$ ) and throughput (by up to 30\%). Finally, we present $R$-Aliph, an implementation of Aliph that is robust, that is, whose performance degrades gracefully in the presence of Byzantine replicas and Byzantine clients.

Categories and Subject Descriptors: C.2.4 [Computer-Communication Networks]: Distributed Systems General Terms: Design, Algorithms, Performance, Fault tolerance

Additional Key Words and Phrases: Abstract, Byzantine fault tolerance, composability, optimization, robustness

\section{ACM Reference Format:}

Pierre-Louis Aublin, Rachid Guerraoui, Nikola Knežević, Vivien Quéma, and Marko Vukolić. 2015. The next 700 BFT protocols. ACM Trans. Comput. Syst. 32, 4, Article 12 (January 2015), 45 pages.

DOI: http://dx.doi.org/10.1145/2658994

\section{INTRODUCTION}

State machine replication (SMR) is a software technique for tolerating failures using commodity hardware. The critical service to be made fault tolerant is modeled by a state machine. Several, possibly different, copies of the state machine are then placed

Authors' addresses: P.-L. Aublin, INSA Lyon, 20 Avenue Albert Einstein, F-69621 Villeurbanne, France; email: pierre-louis.aublin@liris.cnrs.fr; R. Guerraoui, EPFL, LPD (Station 14), CH-1015 Lausanne, Switzerland; email: rachid.guerraoui@epfl.ch; N. Knežević and M. Vukolić (Current address), IBM Research Zurich, Säumerstrasse 4, CH-8803 Rüschlikon, Switzerland; emails: \{kne, mvu\}@zurich.ibm.com; V. Quéma, Grenoble INP / ENSIMAG, 220, rue de la chimie, F-38400 Saint-Martin d'Hères, France; email: vivien.quema@imag.fr.

Permission to make digital or hard copies of part or all of this work for personal or classroom use is granted without fee provided that copies are not made or distributed for profit or commercial advantage and that copies show this notice on the first page or initial screen of a display along with the full citation. Copyrights for components of this work owned by others than ACM must be honored. Abstracting with credit is permitted. To copy otherwise, to republish, to post on servers, to redistribute to lists, or to use any component of this work in other works requires prior specific permission and/or a fee. Permissions may be requested from Publications Dept., ACM, Inc., 2 Penn Plaza, Suite 701, New York, NY 10121-0701 USA, fax +1 (212) 869-0481, or permissions@acm.org.

(c) 2015 ACM 0734-2071/2015/01-ART12 $\$ 15.00$

DOI: http://dx.doi.org/10.1145/2658994 
on different nodes. Clients of the service access the replicas through an SMR protocol, which ensures that, despite contention and failures, replicas perform client requests in the same order.

Two objectives underlie the design and implementation of an SMR protocol: robustness and performance. Robustness conveys the ability to ensure availability (liveness) and one-copy semantics (safety) despite failures and asynchrony. On the other hand, performance measures the time it takes to respond to a request (latency) and the number of requests that can be processed per time unit (throughput). The most robust protocols are those that tolerate (1) arbitrarily large periods of asynchrony, during which communication delays and process-relative speeds are unbounded, and (2) arbitrary (Byzantine) failures of any client, as well as up to one-third of the replicas (this is the theoretical lower bound [Toueg 1984; Lamport 2003]). These are called Byzantine Fault-Tolerant SMR protocols, or simply BFT protocols,${ }^{1}$ for example, PBFT, QU, HQ, Zyzzyva, Spinning, Prime, and Aaardvark [Castro and Liskov 2002; Abd-El-Malek et al. 2005; Cowling et al. 2006; Kotla et al. 2010; Veronese et al. 2009; Amir et al. 2011; Clement et al. 2009]. The ultimate goal of the designer of a BFT protocol is not only to provide robustness to Byzantine faults and asynchrony but also to exhibit comparable performance to a nonreplicated server under "common" circumstances that are considered the most frequent in practice. The notion of "common" circumstance might depend on the application and underlying network, but it usually means network synchrony, rare failures, and sometimes also the absence of contention.

Not surprisingly, even under the same notion of "common" case, there is no "one size fits all" BFT protocol. According to our own experience, the performance differences among the protocols can be heavily impacted by the actual network, the size of messages, the very nature of the "common" case (e.g., contention or not), the actual number of clients, the total number of replicas, and the cost of the cryptographic libraries being used. This echoes Singh et al. [2008], who concluded for instance that "PBFT [Castro and Liskov 2002] offers more predictable performance and scales better with payload size compared to Zyzzyva [Kotla et al. 2010]; in contrast, Zyzzyva offers greater absolute throughput in wider-area, lossy networks." In fact, besides all BFT protocols mentioned earlier, there are good reasons to believe that we could design new protocols outperforming all others under specific circumstances. We do indeed present examples of such protocols in this article.

To deploy a BFT solution, a system designer will hence certainly be tempted to adapt a state-of-the-art BFT protocol to the specific application/network setting, and possibly keep adapting it whenever the setting changes. But this can rapidly turn into a nightmare. All protocol implementations we looked at involve around 20,000 lines of (nontrivial) $\mathrm{C}++$ code, for example, PBFT and Zyzzyva. Any change to an existing protocol, although sometimes algorithmically intuitive, is very painful. Moreover, the changes of the protocol needed to optimize for the "common" case sometimes have strong impacts on the parts of the protocol used in other cases (e.g., "view change" in Zyzzyva). Finally, proving that a BFT protocol is correct is notoriously difficult: the only complete proof of a BFT protocol we knew of is that of PBFT and it involves 35 pages. $^{2}$ This difficulty, together with the impossibility of exhaustively testing distributed protocols [Chandra et al. 2007], would rather plead for never changing a protocol that was widely tested, for example, PBFT.

\footnotetext{
${ }^{1}$ Whereas BFT generally refers to a broader set of Byzantine fault-tolerant techniques, in this article, which focuses on BFT state machine replication (SMR), we use BFT as a shorthand for BFT SMR.

${ }^{2}$ It took Roberto De Prisco a PhD (MIT) to formally prove (using IOA) the correctness of a state machine protocol that does not even deal with Byzantine faults.
} 
We propose in this article a way to have the cake and eat a big chunk of it. We present Abstract (ABortable STate mAChine replicaTion), a new abstraction for designing and reconfiguring generalized state machines that look like traditional state machines with one exception: they may sometimes abort a client's request. Following the divide-and-conquer principle, we then use Abstract to build BFT protocols as compositions of instances of our abstraction, each instance targeted and optimized for specific system conditions.

The progress condition under which an Abstract instance should not abort is a generic parameter. ${ }^{3}$ An extreme instance of Abstract is one that never aborts: this is exactly a traditional (replicated) state machine. Interesting instances are "weaker" ones, in which an abort is allowed, for example, if there is asynchrony or failures (or even contention). When such an instance aborts a client request, it returns a request history that is used by the client (proxy) to "recover" by switching to another instance of Abstract, for example, one with a stronger progress condition. This new instance will commit subsequent requests until it itself aborts. This paves the path to composability and flexibility of BFT protocol design using Abstract. Indeed, the composition of any two Abstract instances is idempotent, yielding another Abstract instance. Hence, and as we will illustrate in the article, the development (design, test, proof, and implementation) of a BFT protocol boils down to:

-Developing individual Byzantine fault-tolerant Abstract instances. This is usually much simpler than tolerating Byzantine faults within a full-fledged, monolithic state machine replication protocol and allows for very effective schemes. A single Abstract instance can be crafted solely with its progress in mind, irrespective of other instances.

-Implementing a switching mechanism to glue together different Abstract instances. This typically involves devising a library that exposes the abort subprotocol of the aborting Abstract instance to the next Abstract instance. In this way, the next Abstract instance is initialized using abort indications of the aborting Abstract instance.

-Ensuring that a request is not aborted by all Abstract instances. This can be made very simple by reusing, as a black box, an existing BFT protocol within one of the instances without indulging in complex modifications.

To demonstrate the benefits of Abstract, we present three BFT protocols, each of which we believe is interesting in its own right:

(1) AZyzzyva, a protocol that illustrates the ability of using Abstract to significantly ease the development of BFT protocols. AZyzzyva is the composition of two Abstract instances: (i) ZLight, which mimics Zyzzyva [Kotla et al. 2010] when there are no asynchrony or failures, and (ii) Backup, which handles the periods with asynchrony/failures by reusing, as a black box, a legacy BFT protocol. We leveraged PBFT, which was widely tested. The code line count to obtain AZyzzyva is, conservatively, around one-third of that of Zyzzyva while keeping the same optimizations (such as batching and read-only request handling). In some sense, had Abstract been identified several years ago, the designers of Zyzzyva would have had a much easier task devising a correct protocol exhibiting the performance they were seeking.

(2) Aliph, a protocol that demonstrates the ability of using Abstract to develop novel efficient BFT protocols. Aliph achieves up to 30\% lower latency and up to $360 \%$

\footnotetext{
${ }^{3}$ Abstract can be viewed as a virtual type; each specification of the progress condition defines a concrete type. These genericity ideas date back to the seminal paper of Landin: The Next 700 Programming Languages (CACM, March 1966).
} 
higher throughput than state-of-the-art protocols. Aliph uses, besides the Backup instance used in $A Z y z z y v a$ (to handle the cases with asynchrony/failures), two new instances: (i) Quorum, targeted for system conditions that do not involve asynchrony/failures/contention, and (ii) Chain, targeted for high-contention conditions without asynchrony/failures. Quorum has a very low latency (like, e.g., Brasileiro et al. [2001], Abd-El-Malek et al. [2005], and Dobre and Suri 2006]), and it makes Aliph the first BFT protocol to achieve a latency of only two message delays with as few as $3 f+1$ servers. Chain implements a pipeline message pattern and relies on a novel authentication technique. It makes Aliph the first BFT protocol with a number of MAC operations at the bottleneck server that tends to 1 in the absence of asynchrony/failures. This contradicts the claim that the lower bound is 2 [Kotla et al. 2010]. Interestingly, each of Quorum and Chain could be developed independently and required less than $35 \%$ of the code needed to develop state-of-the-art BFT protocols. ${ }^{4}$

(3) R-Aliph, a protocol based on Aliph that achieves about the same performance as Aliph in the absence of faults, and that performs significantly better than Aliph under attack, that is, when Byzantine replicas and Byzantine clients act maliciously in order to decrease the performance of the system. Similarly to Aliph, R-Aliph uses the Quorum and Chain protocols when there are no attacks. In order to achieve good performance under attack, R-Aliph monitors the progress of Chain and Quorum, implements various mechanisms to bound the time required to switch from one protocol to another protocol, and uses the Aardvark protocol [Clement et al. 2009] as Backup instance. Aardvark is a BFT protocol that has been specifically designed to sustain good performance despite attacks.

The rest of the article is organized as follows. Section 2 describes the system model. Section 3 presents Abstract. Afterward, we describe our new BFT protocols: AZyzzyva in Section 4, Aliph in Section 5, and R-Aliph in Section 6. Section 7 discusses the related work. Finally, Section 8 discusses future work and concludes the article. Correctness arguments and proofs of our implementations are postponed to Appendix A.

\section{SYSTEM MODEL}

We assume a message-passing distributed system using a fully connected network among processes: clients and servers. The links between processes are asynchronous and unreliable: messages may be delayed or dropped (we speak of link failures). However, we assume fair-loss links: a message sent an infinite number of times between two correct processes will be eventually received. Processes are Byzantine fault prone; processes that do not fail are said to be correct. A process is called benign if it is correct or if it fails by simply crashing. In our algorithms, we assume that any number of clients and up to $f$ out of $n=3 f+1$ servers can be Byzantine. We assume a strong adversary that can coordinate faulty nodes; however, we assume that the adversary cannot violate cryptographic techniques like collision-resistant hashing, message authentication codes (MACs), and signatures.

We further assume that during synchronous periods, there are no link failures, that is, that correct processes can communicate and process messages in a timely manner. More specifically, we assume that during synchronous periods, (1) any message $m$ sent between two correct processes is delivered within a bounded delay $\Delta_{c},(2)$ any message received by a correct process is processed (including possible application-level execution) within a bounded delay $\Delta_{p}$, and (3) $\Delta_{c}$ and $\Delta_{p}$ are known to all correct processes. Intuitively, bounds $\Delta_{c}$ and $\Delta_{p}$ only serve to conveniently define timers that

\footnotetext{
${ }^{4}$ Our code counts are in fact conservative since they do not discount for the libraries shared between $Z$ Light, Quorum, and Chain, which amount to about $10 \%$ of a state-of-the-art BFT protocol.
} 
are assumed not to expire in the "common" case; our BFT protocol constructions from Abstract work correctly in the traditional partially synchronous model [Dwork et al. 1988]. We also denote $\Delta_{p}+\Delta_{c}$ as $\Delta$.

Finally, we declare contention in an Abstract instance whenever there are two concurrent requests such that both requests are invoked but not yet committed/aborted.

\section{ABSTRACT}

In this section, we present our new approach for the design and reconfiguration of abortable state machine replication protocols. We start with an overview of Abstract, the new abstraction we propose. Then, we illustrate the way Abstract can be used to design BFT protocols. Finally, we provide the formal specification of Abstract and state and prove the Abstract composability theorem.

\subsection{Overview}

Abstract has been devised with Byzantine faults in mind, but it is not restricted to them. In fact, Abstract specification does not explicitly refer to any specific kind of faults.

Individually, each Abstract instance behaves just like a (replicated) state machine: it commits clients' requests, returning state-machine-dependent replies to clients. Each reply is a function of a sequence of clients' requests called a commit history [van Renesse and Guerraoui 2010]. As in traditional state machine replication [Schneider 1990], commit histories are totally ordered-we refer to this property of Abstract as Commit Order. However, an Abstract instance needs to commit a request only if certain Progress conditions are met; otherwise, an Abstract instance may abort a client's request. These Progress conditions, determined by the developer of the particular Abstract instance, might depend on the design goals and the environment in which a particular instance is to be deployed. Intuitively, designing a variant of a replicated state machine, in particular a Byzantine fault-tolerant one, that needs to make progress only when certain conditions are met is often considerably simpler than designing a full-fledged replicated state machine that makes progress under all system conditions.

A single Abstract instance is not particularly interesting on its own. That is why the Abstract framework comes with a mechanism and formalism for reconfiguring Abstract instances. Abstract reconfiguration resembles traditional state machine reconfiguration [Lamport et al. 2010; Birman et al. 2010], with the difference that its properties are tailored for reconfiguration of abortable state machines rather than full-fledged, traditional ones. In a sense, Abstract provides a rigorous framework of reconfiguration as an object, using shared-memory style.

More specifically, for the sake of reconfiguration, every Abstract instance has a unique identifier (called instance number) $i$; this instance number abstracts away a set (and a number) of replicas implementing a given instance, the protocol used, possible protocol internals such as a protocol leader, and so forth. For example, in the BFT protocols that we present in this article, Abstract instance number $i$ captures the protocol used and its Progress conditions. Following the classical state machine reconfiguration approach [Lamport et al. 2010; Birman et al. 2010], Abstract reconfiguration can be divided into three steps: (i) stopping the current Abstract instance, (ii) choosing the next Abstract instance, and (iii) combining the request sequences (i.e., commit histories) of separate Abstract instances into a single sequence.

(i) An Abstract instance is automatically stopped as soon as it aborts a single request. This is captured by the Abstract Abort Order property. Namely, when a client's request aborts, along the abort indication, the Abstract instance also returns a sequence of requests called abort history. The Abort Order property mandates that each abort history of a given Abstract instance $i$ contains as its prefix every commit history of instance $i$ (possibly along with some uncommitted requests). In 
a sense, Abstract Abort Order allows a certain request index to become a stopping index-any attempt to order requests higher than this index is guaranteed to abort, effectively causing a given Abstract instance to "stop." As we will see later on, an abort history is used as an input to the next Abstract instance.

(ii) Within an abort indication, an aborting Abstract instance $i$ also returns an identifier of the next instance next $(i)$-we say instance $i$ switches to instance next $(i)$. As in the reconfiguration of classical state machines [Lamport et al. 2010], the Abstract switching requires consensus on the next Abstract instance; hence, we require next to be a function, that is, to have next $(i)$ denote the same instance across all abort indications of instance $i$. Moreover, since we stop aborting instances and to avoid "switching loops," we require monotonically increasing instance numbers, that is, for every instance $i, n \operatorname{ext}(i)>i$.

In the context of the protocols presented in this article, we consider next $(i)$ to be a predetermined function (e.g., known to servers implementing a given Abstract instance); we talk about deterministic or static switching. Concretely, in our protocols, we simply fix next $(i)=i+1$. However, this is not required by our specification; next $(i)$ can be computed "on the fly" by an Abstract implementation (e.g., depending on the current workload or possible failures or asynchrony). In this case, we talk about dynamic switching; this is out of the scope of this article.

(iii) Finally, "state transfer," that is, migration of histories between Abstract instances, reveals critical Abstract subtleties. In short, the client uses the abort history $h$ of an aborting Abstract instance $i$ to invoke next $(i)$; in the context of next $(i), h$ is called an init history. Roughly speaking, init histories are used to initialize instance next $(i)$, before next $(i)$ starts committing/aborting clients' requests.

However, Abstract mandates no explicit agreement on the initial state (i.e., initial sequence of requests) of the next instance next(i). More specifically, there is no agreement across abort histories of an aborting instance $i$. Abort histories need only contain commit histories as their prefix - there is no mutual order imposed on any two abort histories, which are free to contain arbitrary uncommitted requests at their tails.

Similarly, in the context of next $(i)$, no specific init history (recall that init history of instance next $(i)$ is an abort history of instance $i$ ) needs to be a prefix of commit/abort histories of next $(i)$. Abstract only requires implicit agreement on the initial sequence of requests of next $(i)$. This is captured by the Abstract Init Order property, which mandates that, for any Abstract instance, a longest common prefix of init histories is a prefix of any commit/abort history. Intuitively, since every commit history of an aborting instance $i$ is contained as a prefix in every abort history of $i$, we do not need a stronger property than Init Order to maintain total order of commit histories across Abstract instances.

Such weak, implicit ordering of abort/init histories enables very efficient Abstract implementations. Since no explicit agreement is required neither across abort histories nor on the "first" init history, Abstract implementations do not need to solve consensus outside Progress conditions. In fact, and as our Abstract implementations (ZLight, Quorum, and Chain) exemplify, some Abstract specifications with "weak" Progress can be implemented in the asynchronous model despite Byzantine faults, circumventing the FLP impossibility result [Fischer et al. 1985].

With such a design, our Abstract framework has the following appealing properties that simplify the modular and incremental design of state machine replication, and BFT protocols in particular:

(1) Switching between instances is idempotent: the composition of two Abstract instances yields another Abstract instance. 


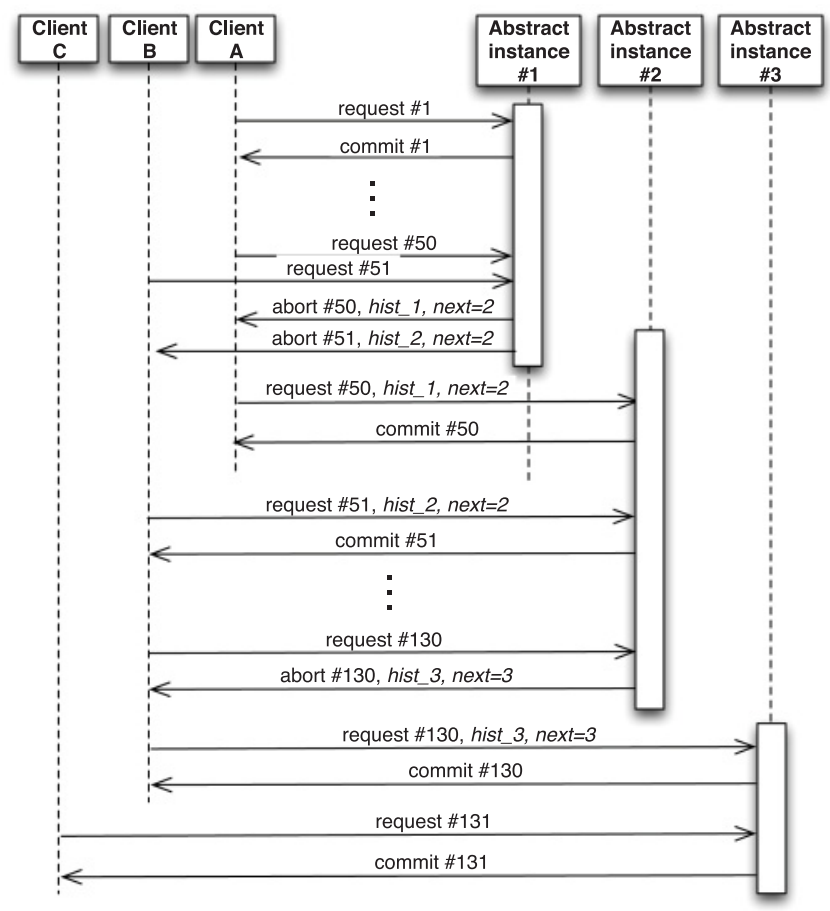

Fig. 1. Abstract operating principle.

(2) A correct implementation of an Abstract instance always preserves the safety of a state machine, that is, the total order across committed requests [Schneider 1990; van Renesse and Guerraoui 2010]. This extends to any composition of Abstract instances.

(3) A (replicated) state machine is nothing but a special Abstract instance—one that never aborts.

Consequently, the designer of a state machine replication (e.g., BFT) protocol has only to make sure that (1) individual Abstract implementations are correct, irrespectively of each other, and (2) the composition of the chosen instances is live: that is, that every request will eventually be committed.

\subsection{Illustration}

Figure 1 depicts a possible run of a BFT protocol built using Abstract. As we have seen before, to preserve consistency, Abstract properties ensure that, at any point in time, only one Abstract instance may commit requests. We say that this instance is active. Client A starts sending requests to the first Abstract instance. The latter commits requests \#1 to \#49, aborts request \#50, and stops committing further requests. Abstract appends to the abort indication an (unforgeable) history (hist_1) and the information about the next Abstract instance to be used (next $=2$ ). Concurrently with request \#50, client B sends request \#51 to the first Abstract instance. Similarly to request \#50, request \#51 aborts and $\mathrm{B}$ obtains an (unforgeable) history hist_2. This abort indication contains the same, previously committed requests \#1 to \#49, just like history hist_1, but appended information on aborted requests differs.

Client A then sends to the new Abstract instance both its uncommitted request (\#50) and the history returned by the first Abstract instance. Instance \#2 gets initialized 
with the given history and commits request \#50. Client B subsequently sends request \#51 together with the history to the second Abstract instance. The latter being already initialized, it simply ignores the history and commits request \#51. The second abstract instance then commits the subsequent requests up to request $\# 130$, which it aborts. Client B uses the history returned by the second abstract instance to initialize the third abstract instance. The latter commits request \#130. Finally, client $\mathrm{C}$ sends request \#131 to the third instance, which commits it. Note that unlike client B, client C directly accesses the currently active instance. This is possible if client $\mathrm{C}$ knows which instance is active, or if all three Abstract instances are implemented over the same set of replicas: replicas can then, for example, "tunnel" the request to the active instance.

\subsection{Specification}

In this section, we provide the specification of Abstract. We model every Abstract instance as a concurrent shared object, where every instance has a unique identifier $i \in \mathbb{N}$ (a natural number). The type (i.e., the set of possible values) of every Abstract instance is a history; a history $h \in H$, where $H=R E Q^{*}$, is a (possibly empty) sequence of requests req $\in R E Q$, where $R E Q$ is a set of all possible requests. More specifically, $R E Q=C \times C M D \times \mathbb{N}$, where $C$ is the set of clients' IDs, and $C M D$ is the set of all possible state machine commands, whereas the third element (a natural number) in a request is called request identifier.

Abstract $i$ exports one operation: $\operatorname{Invoke}_{i}\left(r e q, h_{I}\right)$, where req $\in R E Q$ is a client's request, and $h_{I} \in H$ is an (optional) sequence of requests called init history; we say the client invokes request req (with init history $h_{I}$ ). By convention, when $i=1$, the invocation never contains an init history.

On the other hand, Abstract instance $i$ returns one of the possible two indications to the client invoking req (with $\left.h_{I}\right)$ :

(1) Commit $_{i}\left(r e q, r e p\left(h_{\text {req }}\right)\right)$, where $h_{\text {req }} \in H$ is a sequence of requests called commit history ( of $i$ ) that contains req. Here, $\operatorname{rep}\left(h_{\text {req }}\right)$ represents the output function of the (replicated) state machine; basically, rep $\left(h_{\text {req }}\right)$ represents the replies that the state machine outputs to clients.

(2) $\operatorname{Abort}_{i}\left(\right.$ req,$h_{A}$, next $\left.(i)\right)$, where $h_{A} \in H$ is a sequence of requests called abort history (of $i$ ) and next is a function that returns an integer, where next $(i)>i$.

We say that a client commits or aborts a request req, respectively. In the case of an abort, we also say that instance Abstract $i$ switches to instance next $(i)$. Intuitively, and as detailed later, abort histories of Abstract instance $i$ are prefixed by commit histories of $i$ and are used as init histories in instance next $(i)$.

We model an execution of a concurrent system composed of the set of Abstract instances and clients using traces. ${ }^{5}$ A trace is a finite sequence of Abstract invocation and indication events. A subtrace of a trace $T$ is a subsequence of the events of $T$. An indication matches an invocation if their Abstract instance and request and the client IDs are the same. A (sub)trace $T$ is sequential if it starts with an invocation event and each invocation (except possibly the last) is immediately followed by a matching indication. An instance subtrace, denoted $T \mid i$, of a trace $T$ is the subsequence of all events in $T$ pertaining to Abstract instance $i$. Similarly, a client subtrace, denoted $T \mid c$, of a trace $T$ is the subsequence of all events in $T$ pertaining to client $c$. Client $c$ is $w e l l$ formed in trace $T$ if client subtrace $T \mid c$ of $T$ is sequential and all requests invoked by $c$ in $T$ are unique (i.e., given two requests $r e q_{0}=\left\langle c, c m d_{0}, r i d_{0}\right\rangle$ and $r e q_{1}=\left\langle c, c m d_{1}, r i d_{1}\right\rangle$,

\footnotetext{
${ }^{5}$ Execution traces are sometimes also called execution histories (see, e.g., Herlihy and Wing [1990]). We use the term "traces" to avoid the possible confusion with Abstract commit/abort/init histories.
} 
we have $c m d_{0} \neq c m d_{1}$ or $\left.r i d_{0} \neq r i d_{1}\right)$. We assume all correct clients to be well formed in all traces.

Given trace $T$ and an invocation/indication event $e v \in T$, a history $h$ is called a valid init history (VIH) for Abstract instance $i$ at event $e v$ if and only if $e v$ follows some indication $\operatorname{Abort}_{j}(*, h, i)$ in $T$, for some Abstract instance $j$ (i.e., such that $h$ is an abort history of $j$ and next $(j)=i$ ). Furthermore, req is called a valid init request (VIR) for Abstract instance $i$ if and only if req is invoked with a VIH for $i$ at invocation of req.

Finally, we define requests valid for Abstract instance $i$. In the special case where $i=1$, any invoked request is valid. In case $i>1$, valid requests are (1) VIR requests for $i$ and (2) informally, the requests invoked after instance $i$ is "initialized," that is, after $i$ commits/aborts some VIR request. More formally, given trace $T$, if the invocation of $r e q$ follows an indication of a request $r e q^{\prime}$ in the instance subtrace $T \mid i$ of $T$, where $r e q^{\prime}$ is a VIR for $i$, then $r e q$ is valid for $i$.

In addition, for every instance $i$, if client $c$ is Byzantine, then every request in $\{c\} \times$ $C M D \times \mathbb{N}$ is valid for $i$.

With these definitions, we are ready to state the properties of Abstract instance $i$ (parameterized by a predicate $P_{i}$ that reflects progress). In the following, "prefix" refers to a nonstrict prefix.

(1) (Validity) For any commit/abort event $e v$ and the corresponding commit/abort history $h$ of $i$, no request appears twice in $h$ and every request in $h$ is a valid request for $i$, or an element of a valid init history for $i$ at $e v$.

(2) (Termination) If a correct client $c$ invokes a valid request req, then $c$ eventually commits or aborts req (i.e., $i$ eventually returns a matching indication).

(3) (Progress) If some predicate $P_{i}$ holds, a correct client never aborts a request.

(4) (Init Order) For any commit/abort event $e v$ and the corresponding commit/abort history $h$ of $i$, the longest common prefix of all valid init histories for $i$ at $e v$ is a prefix of $h$.

(5) (Commit Order) Let $h_{r e q}$ and $h_{r e q^{\prime}}$ be any two commit histories of $i$ : either $h_{\text {req }}$ is a prefix of $h_{\text {req }}$ or vice versa.

(6) (Abort Order) Every commit history of $i$ is a prefix of every abort history of $i$.

It is important to note that Abstract is a strict generalization of a state machine. Namely, a state machine is precisely an Abstract instance (with ID $i=1$ ) that never aborts. In this case, the Abort Order and Init Order properties become irrelevant.

\subsection{Abstract Composability}

The key invariant in the Abstract framework is idempotence: a composition of any two Abstract instances is, itself, an Abstract instance. Two Abstract instances are composed by feeding an abort history of a given instance $i$ to instance next $(i)$ as the latter's init history, that is, by having a client that receives $\operatorname{Abort}_{i}\left(r e q, h\right.$, next $\left._{(i)}\right)$ invoke Invoke $_{\text {next }(i)}(r e q, h)$. The abort/init history $h$ is included in a client's $c$ invocation of next $(i)$ only once, with the first invocation of instance next $(i)$ by $c$. Intuitively, the composed instance can typically have a different Progress property than the original two but is never "weaker" than any of them.

More precisely, consider two Abstract instances: instance 1 that switches to $i$ (denoted, for clarity, by $1 \rightarrow i$ ), and instance $i$ that switches to $j$ (denoted by $i \rightarrow j$ ). Using these two instances, we can implement a single Abstract instance that inherits instance number 1 and switches to $j$ (denoted by $1 \rightarrow j$ ). This implementation is a simple client-side protocol that we call Abstract composition protocol (ACP), which proceeds as follows.

Initially, with each invocation of the implemented instance $1 \rightarrow j\left(\operatorname{Invoke}_{1 \rightarrow j}(r e q)\right)$, a client simply invokes instance $1 \rightarrow i$ Invoke $\left._{1 \rightarrow i}(r e q)\right)$. If a client receives 
Commit $_{1 \rightarrow i}\left(\right.$ req,$\left.h_{\text {req }}\right)$, it outputs Commit $_{1 \rightarrow j}\left(\right.$ req,$\left.h_{\text {req }}\right)$. If, however, a client in ACP receives Abort $_{1 \rightarrow i}(r e q, h, i)$ for the first time, it immediately feeds $h$ to instance $i \rightarrow j$ with $\operatorname{Invoke}_{i \rightarrow j}(r e q, h)$, without "exposing" the Abort indication. From this point on, for every following invocation of the implemented instance $1 \rightarrow j$ (Invoke I $_{\rightarrow j}($ req $)$ ), a client simply invokes Invoke $_{i \rightarrow j}(r e q)$ and never invokes again $1 \rightarrow i$. Finally, for every received Commit $t_{i j}\left(r e q, h_{\text {req }}\right)$, a client outputs $\operatorname{Commit}_{1 \rightarrow j}\left(r e q, h_{\text {req }}\right)$, and for every $\operatorname{Abort}_{i \rightarrow j}\left(r e q, h_{A}, j\right)$, a client outputs $\operatorname{Abort}_{1 \rightarrow j}\left(r e q, h_{A}, j\right)$.

Abstract composability is captured by the following theorem:

Theorem 3.1 [Abstract Composability Theorem]. Given Abstract instance 1 that switches to $i$ and instance $i$ that switches to $j$, the ACP protocol implements Abstract instance 1 that switches to $j$.

In the following, we prove Theorem 3.1, which, by induction, extends to Abstract compositions of arbitrary length. We have also specified Abstract and ACP in TLA+/PlusCal [Lamport 2009] and model-checked the Abstract composability theorem using the TLC model checker. The details are available in the technical report [Guerraoui et al. 2008].

PRoof. In the following, we prove the Abstract properties of instance $1 \rightarrow j$. Notice that Init Order is not relevant for instances with instance number 1 , including $1 \rightarrow j$. We, however, use Init Order of instance $i \rightarrow j$ to prove the other properties of instance $1 \rightarrow j$. We denote the set of commit (resp., abort) histories of Abstract instance $x$ by $\mathrm{CH}_{x}$ (resp., $\mathrm{AH}$ ).

We first prove that individual commit/abort indications of Abstract instance $1 \rightarrow j$ conform to the specification. For commit indications, it is straightforward to see from ACP that $\mathrm{CH}_{1 \rightarrow j}=\mathrm{CH}_{1 \rightarrow i} \cup \mathrm{CH}_{i \rightarrow j}$. Since $\mathrm{CH}_{1 \rightarrow i} \subset \mathrm{H}$ and $\mathrm{CH}_{i \rightarrow j} \subset \mathrm{H}$ (by specifications of $1 \rightarrow i$ and $i \rightarrow j$ ), this implies that for every request req committed by instance $1 \rightarrow j$, its commit history $h_{\text {req }} \in C H_{1 \rightarrow j}$ is in $H$, such that req $\in h_{\text {req }}$. Similarly, it is easy to see that $A H_{1 \rightarrow j}=A H_{i \rightarrow j} \subset H$. Moreover, recall that Abstract instance numbers should monotonically increase; in this case, $1<j$ follows directly from $1<i$ and $i<j$, by specifications of Abstract instances $1 \rightarrow i$ and $i \rightarrow j$.

To prove Validity, observe that no request appears twice in any commit/abort history in $\mathrm{CH}_{1 \rightarrow j} \cup A H_{1 \rightarrow j}$, since, by Validity of $1 \rightarrow i$ and $i \rightarrow j$, no request appears twice in $\mathrm{CH}_{1 \rightarrow i} \cup \mathrm{CH}_{i \rightarrow j} \cup A H_{i \rightarrow j}$. Moreover, every request in every commit/abort history of $1 \rightarrow j$ is an invoked request or a request by a Byzantine client-since the ID of Abstract instance $1 \rightarrow j$ is equal to 1 , all such requests are by definition valid.

Termination also follows directly from Termination of instances $1 \rightarrow i$ and $i \rightarrow j$. Notice that a request req that a correct client $c$ executing ACP invokes on $i \rightarrow j$ without an init history (i.e., such that req is not a VIR for $i \rightarrow j$ ) is indeed valid for $i \rightarrow j$ (and hence the invocation of $r e q$ terminates); namely, before $c$ invokes req, $c$ already received an indication for its VIR invocation of $i \rightarrow j$. Indeed, by ACP, the first invocation of $i \rightarrow j$ is a VIR invocation that contains a valid init history, and, by well formedness of $c, c$ does not invoke $r e q$ before it receives the indication matching its first invocation.

To prove Commit Order, we focus on the case where req and $r e q^{\prime}$ are originally committed by different instances; that is, we focus on showing that for any $h_{\text {req }} \in \mathrm{CH}_{1 \rightarrow i}$ and $h_{r e q^{\prime}} \in C_{i \rightarrow j}, h_{r e q}$ is a prefix of $h_{r e q^{\prime}}$-other cases directly follow from the Commit Order properties of instances $1 \rightarrow i$ and $i \rightarrow j$.

By definition, every VIH for instance $i \rightarrow j$ is an abort history of instance $1 \rightarrow i$. By Abort Order of $1 \rightarrow i$, every commit history of $1 \rightarrow i$, including $h_{\text {req }}$, is a prefix of any abort history of $1 \rightarrow i$. Hence, $h_{\text {req }}$ is a prefix of every VIH for instance $i \rightarrow j$. By Init Order of $i \rightarrow j$, the longest common prefix of VIHs of instance $i \rightarrow j$ is a prefix of all histories in $\mathrm{CH}_{i \rightarrow j} \cup A H_{i \rightarrow j}$, including $h_{r e q^{\prime}}$. This implies that $h_{r e q}$ is a prefix of $h_{r e q}$.

The proof of Abort Order follows the same reasoning as the previous proof of Commit Order. 
Finally, we show that the Progress of $1 \rightarrow j$ holds for some predicate $P_{1 \rightarrow j}$, which, intuitively, is not "weaker" than any of the respective predicates $P_{1 \rightarrow i}$ and $P_{i \rightarrow j}$. We do not attempt, however, to express $P_{1 \rightarrow j}$ in terms of $P_{1 \rightarrow i}$ and $P_{i \rightarrow j}$.

If $P_{1 \rightarrow i}$ holds during an entire execution, clearly $1 \rightarrow j$ never aborts at a correct client as $1 \rightarrow i$ never aborts. On the other hand, if $P_{i \rightarrow j}$ holds during an entire execution, instance $1 \rightarrow i$ might abort the request; however, a correct client executing ACP does not output these abort indications, but immediately invokes instance $i \rightarrow j$. On the other hand, since $P_{i \rightarrow j}$ holds, instance $i \rightarrow j$ never aborts a correct client's request.

To summarize, ACP involves Abstract switching through clients, who receive an abort indication containing an abort history and invoke the next instance. This approach allows for a streamlined specification of Abstract that does not reason about any specific process beyond clients. Indeed, in the Abstract specification, we did not have to mention replicas implementing Abstract, nor any other process apart from clients. This sharedmemory style of Abstract is intentional. In the remainder of this section, we briefly discuss some of the aspects of Abstract composability and its proof.

Alternatives to switching through clients. In practice, due to, for example, performance or security concerns, a need may arise for switching through other processes in the system, beyond clients. For example, it might be useful to switch through a replica implementing Abstract, for example, to save on bandwidth, or through a dedicated reconfigurator process to, for example, (logically) centralize management. One way of achieving this would be to generalize the Abstract specification to accommodate for this. Notice here that our proof of Theorem 3.1 relies on clients only for Progress and Termination to ensure that the state is actually transferred to the next Abstract instance. Instead, for example, the Abstract specification could be generalized so that one or more processes or reconfigurators receive the full abort indication with abort histories instead of a client. Formalizations of such generalizations of our specification are, however, out of the scope of this article.

That said, it is perfectly possible to achieve switching through replicas and still reap benefits of the composability proofs we present here, without introducing new processes to the Abstract specification. To this end, it is sufficient to allow a replica implementing Abstract to act as a client of Abstract by invoking a special noop request that does not modify the state of the replicated state machine. That way, the replica can perform switching itself. ${ }^{6}$ This can be further complemented by disallowing application clients from transferring the state themselves. Such switching through replicas is illustrated in $R$-Aliph, our robust BFT protocol that we present in Section 6.3. Before that, we also discuss an important state transfer performance optimization used in all of our Abstract implementations in Section 4.4.

Byzantine clients. Abstract composability theorem (ACT, Theorem 3.1) holds despite Byzantine clients. Whereas Byzantine clients are not very prominent in our proof of the ACT, notice that this is only so because ACT relies on the composition of implementations of individual Abstract instances $1 \rightarrow i$ and $i \rightarrow j$ that are assumed to be correct despite Byzantine clients. For example, a correct implementation of instance $i \rightarrow j$ must guarantee that a Byzantine client cannot successfully forge an init history; that is, correct implementations of $1 \rightarrow i$ and $i \rightarrow j$ must ensure that any valid init history $h$ for $i \rightarrow j$ was indeed previously output as an abort history of $1 \rightarrow i$. How specific Abstract implementations achieve this causal dependency has no effect on the correctness of ACT; however, in the rest of this article, we will demonstrate how such

\footnotetext{
${ }^{6}$ Of course, in general, depending on Progress of a specific Abstract instance $i$, up to $f+1$ of such replica noop invocations might be required to actually perform switching (e.g., if instance $i$ guarantees Progress despite $f$ Byzantine replicas).
} 


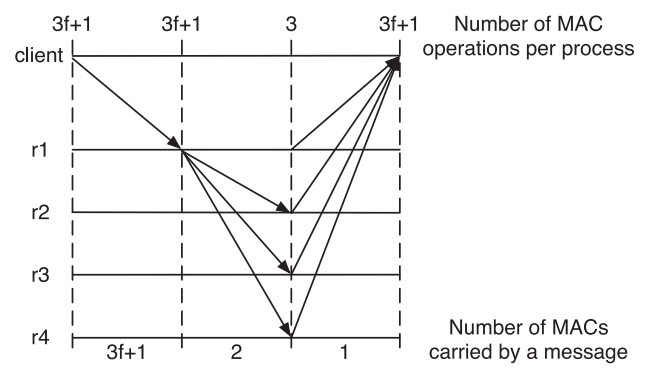

Fig. 2. Communication pattern of ZLight (fast path of Zyzzyva).

correct implementations can be achieved. For example, our implementations use unforgeable digital signatures to enforce causality between abort histories and init histories.

\section{SIMPLE ILLUSTRATION: AZYZZYVA}

In this section, we illustrate how Abstract significantly eases the design, implementation, and proof of BFT protocols. We describe AZyzzyva, a full-fledged BFT protocol that mimics Zyzzyva [Kotla et al. 2010] in its "common case" (i.e., when there are no link or server failures). In "other cases," AZyzzyva relies on Backup, an Abstract implementation with strong progress guarantees that can be implemented on top of any existing $\mathrm{BFT}$ protocol. We chose to mimic Zyzzyva for it is known to be efficient yet very difficult to implement [Clement et al. 2009]. Using Abstract, we had to write and test less than $30 \%$ of the Zyzzyva code to obtain AZyzzyva. Abstract also considerably simplified the proof of AZyzzyva compared to that of Zyzzyva, due to the composability of Abstract instances and the straightforward (and reusable) proof of the Backup module.

We start this section with an overview of AZyzzyva. We then describe the Abstract instances it relies on. Finally, we provide a qualitative assessment, as well as a performance evaluation of $A Z y z z y v a$.

\subsection{Protocol Overview}

As mentioned before, AZyzzyva is a BFT protocol that mimics Zyzzyva [Kotla et al. 2010]. Zyzzyva requires $3 f+1$ replicas. It works as follows. In the "common case," Zyzzyva executes the fast speculative path depicted in Figure 2. A client sends a request to a designated server, called primary ( $r_{1}$ in Figure 2$)$. The primary appends a sequence number to the request and broadcasts the request to all replicas. Each replica speculatively executes the request and sends a reply to the client. All messages in the this sequence are authenticated using MACs rather than (more expensive) digital signatures. The client commits the request if it receives the same reply from all $3 f+1$ replicas.

When a client does not receive the same reply from all $3 f+1$ replicas, Zyzzyva executes a second phase. This second phase thus aims at handling the case with link/server/client failures ("worst case"). Roughly, this phase (that AZyzzyva avoids to mimic) consists of considerable modifications to PBFT [Castro and Liskov 2002], which arise from the "profound effects" [Kotla et al. 2010] that the Zyzzyva "common case" optimizations have on PBFT's "worst case."

Our goal when building AZyzzyva using Abstract is to show that we can completely separate the concerns of handling the "common case" and the "worst case." AZyzzyva uses two different Abstract implementations: ZLight and Backup. Roughly, ZLight is an Abstract that guarantees progress in the Zyzzyva "common case." On the other hand, Backup is an Abstract with strong progress: it is guaranteed to commit an exact 


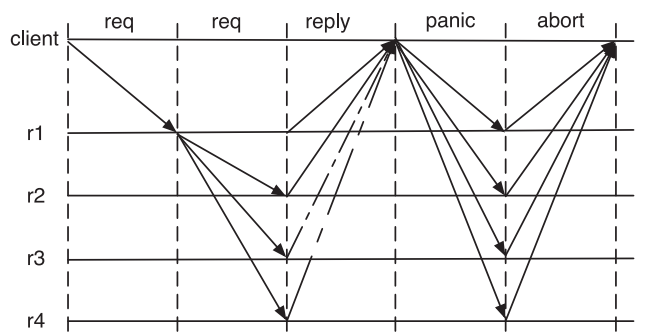

Fig. 3. Communication pattern of ZLight outside the "common case" (the three first rounds correspond to the "common case").

certain number of requests $k$ ( $k$ is itself configurable) before it starts aborting. AZyzzyva works as follows: every odd (resp., even) Abstract instance is ZLight (resp., Backup). This means that ZLight is first executed. When it aborts, it switches to Backup, which commits the next $k$ requests. Backup then aborts subsequent requests and switches to (a new instance of) ZLight, and so on.

In the following, we describe ZLight and Backup. Then, we assess the qualitative benefit of using Abstract. Finally, we discuss the performance of AZyzzyva.

\subsection{ZLight}

ZLight implements Abstract with the following progress property that reflects Zyzzyva's "common case": it commits requests when (1) there are no server or link failures, and (2) no client is Byzantine (crash failures are tolerated). When this property holds, ZLight implements Zyzzyva's "common case" pattern (Figure 2), described earlier. Outside the "common case," that is, when a client does not receive $3 f+1$ consistent replies, ZLight performs additional steps, depicted in Figure 3 (see the last two communication steps). The client sends a PANIC message to replicas. Upon reception of this message, replicas stop executing requests and send back a signed abort message containing their history (replicas will now send the same abort message for all subsequent requests). When the client receives $2 f+1$ signed messages containing replica histories, it can generate an abort history. It will then use this abort history to switch to Backup.

In the remainder of this section, we give the pseudo-code of ZLight. We first present the code executed in the common case. For ease of presentation, we do not mention init histories. We then present the pseudo-code executed when ZLight aborts, followed by a presentation of the pseudo-code executed to initialize a new ZLight instance. Finally, we conclude by presenting the pseudo-code of a checkpointing protocol that is used in ZLight to garbage collect some data stored by replicas.

4.2.1. Common Case. This section describes the pseudo-code executed by ZLight in the common case. A message $m$ sent by a process $p$ to a process $q$ and authenticated with a MAC is denoted by $\langle m\rangle_{\mu_{p . q}}$. A process $p$ can use vectors of MACs (called authenticators [Castro and Liskov 2002]) to simultaneously authenticate a message $m$ for multiple recipients belonging to a set $S$; we denote such a message, which contains $\langle m\rangle_{\mu_{p, q}}$ for every $q \in S$, by $\langle m\rangle_{\alpha_{p, S}}$. In addition, we denote the digest of a message $m$ by $D(m)$, and $\langle m\rangle_{\sigma_{p}}$ denotes a message that contains $D(m)$ signed by the private key of process $p$ and the message $m$. Notations for message fields and client/replica local variables are shown in Figure 4. To help distinguish clients' requests for the same command $o$, we assume that client $c$ calls $\operatorname{Invoke}_{i}(r e q)$, where $r e q=\left\langle c, o, t_{c}\right\rangle$ and where $t_{c}$ is a unique, monotonically increasing client's timestamp. A replica $r_{j}$ logs req by appending it to its local history, denoted $L H_{j}$. A replica $r_{j}$ executes req by applying it to the state of the 


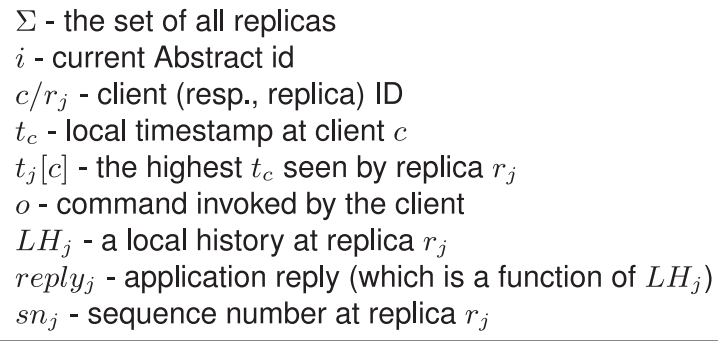

Fig. 4. Message fields and local variables.

replicated state machine and by calculating an application-level reply (i.e., the reply of a replicated state machine) for the client.

We detail here the various steps that are executed when a client invokes a request. For the sake of brevity, we omit to mention that upon receiving a message $m$, a process $p$ first checks that $m$ has a valid authenticator and does not process the message if that is not the case.

Step Z1. On Invoke $_{i}($ req $)$, client $c$ sends a message $m^{\prime}=\langle\mathrm{REQ}, \text { req, } i\rangle_{\alpha_{c, \Sigma}}$ to the primary (say, $r_{1}$ ) and triggers timer $T$ set to $3 \Delta$.

Step Z2. On receiving $m^{\prime}=\langle\operatorname{REQ}, \text { req, } i\rangle_{\alpha_{c, \Sigma}}$, if req.t. is higher than $t_{1}[c]$, then the primary $r_{1}$ (i) updates $t_{1}[c]$ to req.t. $t_{c}$, (ii) increments $s n_{1}$, and (iii) sends $\left\langle\langle\text { ORDER, req, } i, s n\rangle_{\mu_{r_{1}, r_{j}}}, M A C_{j}\right\rangle$ to every replica $r_{j}$, where $M A C_{j}$ is the MAC entry for $r_{j}$ in the client's authenticator for $m^{\prime}$.

Step Z3. On receiving (from primary $r_{1}$ ) 〈 $\left.\left\langle\text { ORDER, req, } i, s n^{\prime}\right\rangle_{\mu_{r_{1}, r_{j}}}, M A C_{j}\right\rangle$, if (i) $M A C_{j}$ authenticates $r e q$ and $i$, (ii) $s n^{\prime}=s n_{j}+1$, and (iii) $t_{j}[c]<$ req.t $t_{c}$, then replica $r_{j}$ (i) updates $s n_{j}$ to $s n^{\prime}$ and $t_{j}[$ req.c] to req.t. $\left\langle\text { RESP, reply } y_{j}, D\left(L H_{j}\right), i \text {, req. } t_{c}, r_{j}\right\rangle_{\mu_{r j}, c}$ to $c .{ }^{7}$ If $M A C_{j}$ verification fails, $r_{j}$ stops executing Step Z3 in instance $i$.

Step Z4. If client $c$ receives $3 f+1$ 〈RESP, reply, LHDigest, $i$, req. $\left.t_{c}, *\right\rangle_{\mu_{* c}}$ messages from different replicas before expiration of $T$, with identical digests of replicas' local history (LHDigest) and identical replies (or digests thereof), then the client commits req with reply. Otherwise, the client triggers the panicking mechanism as explained in Section 4.2.2 (Step P1).

4.2.2. Aborting. In this section, we describe the pseudo-code of the panicking mechanism that clients trigger when they do not receive $3 f+1$ consistent replies (in Step Z4). As soon as the execution of this mechanism completes, the client is able to generate an abort history that it can use to switch to another Abstract instance (Backup in the case of the AZyzzyva protocol).

Step P1. If the client does not commit request req by the expiration of timer $T$ (triggered in Step Z1), $c$ panics; that is, it sends a $\left\langle\text { PANIC, req. } t_{c}\right\rangle_{\mu_{c, r_{j}}}$ message to every replica $r_{j}$. Since messages may be lost, the client periodically sends PANIC messages until it aborts the request.

Step P2. Replica $r_{j}$, on receiving a $\left\langle\text { PANIC, req. } t_{c}\right\rangle_{\mu_{c, r_{j}}}$ message, stops executing new requests (i.e., stops executing Step Z3) and sends $\left\langle\text { ABORT, req. } t_{c}, L H_{j} \text {, next }(i)\right\rangle_{\sigma_{r_{j}}}$ to $c$ (with periodic retransmission).

\footnotetext{
${ }^{7}$ Here (see also Figure 4), reply $y_{j}=\operatorname{rep}\left(L H_{j}\right)$, where rep is the function within a replicated state machine (i.e., application) that computes the reply to the client. As an optimization, all but one designated replica can send reply digests $D\left(r e p l y_{j}\right)$ instead of reply $y_{j}$ within a RESP message.
} 
Step P3. When client $c$ receives $2 f+1\left\langle\text { ABORT, req. } t_{c}, *, n e x t(i)\right\rangle_{\sigma_{*}}$ messages with correct signatures from different replicas and the same value for next $(i)$, the client collects these messages into a set $\operatorname{Proof}_{A H_{i}}$ and extracts the abort history $A H_{i}$ from $\operatorname{Proof}_{A H_{i}}$ as follows: first, $c$ generates history $A H$ such that $A H[j]$ equals the value that appears at position $j \geq 1$ of $f+1$ different histories $L H_{j}$ that appear in $\operatorname{Proof}_{A H_{i}}$; if such a value does not exist for position $x$, then $h$ does not contain a value at position $x$ or higher. Then, $c$ extracts the abort history $A H_{i}$ by taking the longest prefix of $A H$ in which no request appears twice.

4.2.3. Initializing a ZLight Instance. In this section, we describe the way a ZLight instance is initialized. We assume that a client extracted an abort history $A H_{i}$ and that it invokes instance $i^{\prime}=n e x t(i)$. To do so, it accompanies req with init history $I H_{i^{\prime}}=A H_{i}$ and $\operatorname{Proof}_{\mathrm{AH}_{i}}$. We summarize here the additional actions performed by processes in the various steps of ZLight to take into account init histories.

Step Z1+. On Invoke $_{i^{\prime}}(r e q, I H)$, the message(s) sent by the client also contains $I H$ and the set of signed ABORT messages Proof $f_{I H}$ returned by the preceding Abstract $i$, where $i^{\prime}=\operatorname{next}(i)$.

Step Z2+. If its local history $L H_{1}$ is empty, the primary/head $r_{1}$ executes the step only if (i) $I H$ can be verified against Proof $f_{I H}$, following the algorithm given in Step P3 (Section 4.2.2), and (ii) ABORT messages in Proof $f_{A H_{i}}$ indeed declare $i^{\prime}$ as next $(i)$.

Step Z3+. If its local history $L H_{j}$ is empty, the replica $r_{j}$ executes the step only if (i) $I H$ can be verified against Proof $f_{I H}$, following the algorithm given in Step P3 (Section 4.2.2), and (ii) ABORT messages in $\operatorname{Proof}_{A H_{i}}$ indeed declare $i^{\prime}$ as next(i). If so, then (before executing $r e q$ ) $r_{j}$ logs all the requests contained in $I H$ (i.e., $r_{j}$ sets $L H_{j}$ to $I H)$; then $r_{j}$ logs req unless $r e q$ was already in $I H$.

Step P1+. On sending PANIC messages for a request that was invoked with an init history, the client also includes $I H$ and the set of signatures Proof $f_{I H}$ returned by the preceding Abstract $i$ within a PANIC message.

Step P2+. If its local history $L H_{j}$ is empty, replica $r_{j}$ executes the step only if (i) IH can be verified against Proof $f_{H H}$, following the algorithm given in Step P3 (Section 4.2.2), and (ii) ABORT messages in Proof $f_{A H_{i}}$ indeed declare $i^{\prime}$ as next $(i)$. Then, before executing the step as described in Section 4.2.2, $r_{j}$ first sets $L H_{j}$ to $I H$.

4.2.4. Checkpointing. ZLight uses a lightweight checkpoint subprotocol (LCS) to truncate histories every $C H K$ requests (in our performance evaluation, $C H K=128$ ). LCS is very similar to checkpoint protocols used in Castro and Liskov [2002] and Kotla et al. [2010]. Its operating principle is the following:

(1) Every replica $r_{j}$ increments a checkpoint counter $c c$ and sends it along with the digest of its local state to every other replica (using simple point-to-point MACs), when its (noncheckpointed suffix of) local history reaches $C H K$ requests. Then, $r_{j}$ triggers a checkpoint timer.

(2) If the timer expires and there is no checkpoint, the replica stops executing all requests and retransmits its last checkpoint message to every other replica.

(3) If replica $r_{j}$ receives the digest of the same state $s t_{c c}$ with the same checkpoint counter number $c c$ greater than lastcc (initially lastcc $=0$ ) from all replicas, $r_{j}$ (a) truncates its local history and checkpoints its state to $s t_{c c}$ and (b) stores $c c$ to variable lastcc. Checkpointed state $s t_{c c}$ becomes a prefix of replicas' local histories to which new requests are appended and is treated as such in all operations on local histories in our algorithms. Moreover, every abort or commit history of length at most $c c * C H K$ is considered to be a prefix of $s t_{c c}$.

With LCS, Step P2 of the panicking mechanism (Section 4.2.2) is modified so that a digest of the last checkpointed state, the last checkpoint number, and the 
noncheckpointed suffix of the local history are propagated by an aborting replica $r_{j}$ in place of a complete local history $L H_{j}$. Note, however, that LCS has an additional slight impact on the panicking/aborting mechanism. Consider, for example, replicas performing a checkpoint $c c=34$ concurrently with some client panicking. What may happen is that when the PANIC message is received by, say, replicas $r_{1}$ and $r_{2}$, the local state of replica $r_{1}$ is already truncated to $s t_{34}$, whereas the local state of replica $r_{2}$ is still $s_{33}$ followed by $C H K$ requests. We refer to this effect as partial checkpoint. If $r_{1}$ and $r_{2}$ send such (seemingly different) information to the panicking client within ABORT messages, a client might not be able to tell that the local states and local histories of replicas $r_{1}$ and $r_{2}$ are, in fact, identical. This may in turn impact the panicking/aborting subprotocol as already described. To this end, when (a) the noncheckpointed suffix of local history of replica $r_{j}$ contains $C H K$ requests or more, but a replica $r_{j}$ did not yet perform a checkpoint $c c$, and (b) replica $r_{j}$ is about to send an ABORT message, then replica $r_{j}$ tentatively performs checkpoint $c c$ and calculates $s t_{c c}$ prior to sending an ABORT. Then, $r_{j}$ adds the digest of $s t_{c c}$ to its ABORT message that already contains the digest of $s t_{c c-1}$ and the noncheckpointed suffix, as explained earlier. This allows the client to avoid the apparent ambiguity in identifying the same histories in Step P3, Section 4.2.2, in case of a partial checkpoint.

\subsection{Backup}

Backup is an Abstract implementation with a progress property that guarantees that exactly $k \geq 1$ requests will be committed, where $k$ is a generic parameter (we explain our configuration for $k$ at the end of this section). We employ Backup in AZyzzyva to ensure progress outside "common cases" (e.g., under replica failures).

We implemented Backup as a very thin wrapper (around 600 lines of $\mathrm{C}++$ code) that can be put around any existing BFT protocol. In our $\mathrm{C} / \mathrm{C}++$ implementations, Backup is implemented over PBFT [Castro and Liskov 2002], for PBFT is the most extensively tested BFT protocol and it is proven correct. Other existing BFT protocols that provide robust performance under failures, like Aardvark [Clement et al. 2009], are also very good candidates for the Backup basis, as illustrated in Section 6.3.

To implement Backup, we exploit the fact that any BFT protocol can totally order requests submitted to it and implement any functionality on top of this total order. In our case, Backup is precisely this functionality. Backup works as follows: it ignores all the requests delivered by the underlying BFT protocol until it receives a request containing a valid init history, that is, an unforgeable abort history generated by the preceding Abstract (ZLight in the case of AZyzzyva). At this point, Backup sets its state by executing all the requests contained in the valid init history it received. Then, it simply executes the first $k$ requests ordered by the underlying BFT protocol (neglecting subsequent init histories) and commits these requests. After committing the $k^{\text {th }}$ request, Backup aborts all subsequent requests, returning the signed sequence of executed requests as the abort history. A client can switch from Backup as soon as it receives $f+1$ signed messages from different replicas, containing an identical abort history and the same next Abstract instance ID $i^{\prime}$. This is a reasonable requirement on the BFT protocol that underlies Backup, since any BFT protocol must anyway provide an identical reply from at least $f+1$ replicas; in the case of Backup abort history, we just require this particular reply to be signed (all existing BFT protocols we know of provide a way to digitally sign messages).

The parameter $k$ used in Backup is generic and is an integral part of the Backup progress guarantees. Our default configuration increases $k$ exponentially, with every new instance of Backup. This ensures the liveness of the composition, which might not 
be the case with, say, a fixed $k$ in a corner case with very slow clients. ${ }^{8}$ More importantly, in the case of failures, we actually do want to have a Backup instance remaining active for long enough, since Backup is precisely targeted to handle failures. On the other hand, to reduce the impact of transient link failures, which can drive $k$ to high values and thus confine clients to Backup for a long time after the transient failure disappears, we flatten the exponential curve for $k$ to maintain $k=1$ during some targeted outage time. ${ }^{9}$ In our implementation, we also periodically reset $k$. Dynamically adapting $k$ to fit the system conditions is appealing but requires further studies and is out of the scope of this article.

\subsection{State Transfer Optimization}

Sending entire local histories to clients within ABORT messages in Step P2 of the panicking/aborting subprotocol (Section 4.2.2) might be expensive, even if local histories are checkpointed as described in Section 4.2.4. To this end, all our Abstract implementations presented in this article, except Backup, implement the following state transfer optimization when switching to the next Abstract instance. ${ }^{10}$

Upon receiving a PANIC message from a client in Step P2, replica $r_{j}$ sends an ABORT message to the client containing the signed history of digests of (noncheckpointed) requests in place of the signed history of (noncheckpointed) requests. The client performs Step P3 normally, except that it extracts an abort history that contains digests of requests rather than an abort history that contains full requests.

A client normally uses such an abort history (together with the corresponding proof of $2 f+1$ ABORT messages) as an init history of the next Abstract instance. During initialization of the next instance, the replicas verify the abort history normally. Often, a replica being initialized will have all the requests locally and will initialize its local state accordingly. However, a replica might not have some requests whose digests appear in the init history-we speak of missing requests. If a replica misses requests at initialization, it simply asks other replicas for inter-replica state transfer of missing requests. This procedure is guaranteed to terminate since each digest of the request in the init history is vouched for by at least $f+1$ replicas; for each request, at least one of these replicas is correct and supplies the missing request.

\subsection{Qualitative Assessment}

In evaluating the effort of building AZyzzyva, we focus on the cost of ZLight in terms of lines of code. Indeed, Backup, for which the additional effort is small (around 600 lines of $\mathrm{C}++$ code), can be reused for other BFT protocols in our framework. For instance, we use Backup in our Aliph and R-Aliph protocols as well (Sections 5 and 6).

Our main observation is that it took 4,086 lines of code to implement ZLight, in contrast to 14,339 of lines of code needed for Zyzzyva. Note that we implemented and used an external library that contains cryptographic functions, networking code (to send/receive messages and manage sockets), and data structures (e.g., maps, sets).

\footnotetext{
${ }^{8}$ In short, $k$ requests committed by a single Backup instance $i$ might all be invoked by the same, fast client. A slow client can then get its request aborted by $i$. The same can happen with a subsequent Backup instance, and so forth. This issue can be avoided by exponentially increasing $k$ (for any realistic load that does not increase faster than exponentially) or by having the replicas across different Abstract instances share a client input buffer.

${ }^{9}$ For example, using $k=\left\lceil C * 2^{m}\right\rceil$, where $m$ is incremented with every new Abstract instance, with the rough average time of $50 \mathrm{~ms}$ for switching between two consecutive Backup instances in AZyzzyva, we can maintain $k=1$ during $10 s$ outages with $C=2^{-200}$.

${ }^{10}$ In Backup, to minimize the size of state transfer through clients, we simply align switching with a checkpoint, since the former can be performed at will.
} 
This library roughly contains 7,500 lines of code that are not taken into account. To provide a fair comparison, we ported Zyzzyva on this library. In other words, to build ZLight, we needed less than $30 \%$ of the Zyzzyva line count (14,339 lines).

This line-of-code comparison has to be taken with a grain of salt: (1) these protocols have been developed and proved by different researchers, and (2) Zyzzyva does not fully implement the code required to handle faults. Yet, we believe that the line-of-code metric provides a useful intuition of the difference in code and algorithmic complexity between Zyzzyva and ZLight since both implementations use the same code base, inherited from PBFT [Castro and Liskov 2002].

Another benefit of using Abstract is the fact that we did not have to prove from scratch the correctness of AZyzzyva in all possible executions. Namely (see also Appendix A), to prove $A Z y z z y v a$, we needed to concentrate our effort only on proving the correctness of ZLight. The general Abstract composability Theorem 3.1 and the straightforward proof of the reusable Backup module complement the entire proof.

Finally, it is fair to note that incremental development using Abstract may, in fact, increase the code base that needs to be maintained. Indeed, in our case, instead of maintaining only Zyzzyva or PBFT, we would need to maintain both ZLight and PBFT/Backup, which together have more lines of code than any of Zyzzyva or PBFT individually. In this sense, there is no free lunch: adaptive performance of Abstract has a certain price with respect to monolithic BFT protocols. While more lines of code might seemingly imply more vulnerabilities for Byzantine attacks and more maintenance effort, we believe that this is not the case due to simplified design, proofs, and testing of individual modules. Such modular designs are widely used in traditional software engineering, where monolithic solutions that might have fewer lines of code often have more bugs and are more difficult to maintain.

\subsection{Performance Evaluation}

We have compared the performance of AZyzzyva and Zyzzyva in the "common case" using the benchmarks described in Section 5.4. Not surprisingly, AZyzzyva and Zyzzyva have identical performance in this case, since in the "common case" the two protocols are the same. In this section, we do thus focus on the cost induced by our switching mechanism when the operating conditions are outside the common case (and ZLight aborts a request).

To assess the switching cost, we perform the following experiments: we feed the request history of $Z$ Light with $r$ requests of size $1 \mathrm{kB}$. We then issue 10,000 successive requests. To isolate the cost of the switching mechanism, we do not execute the $Z$ Light common case; the measured time consists of the time required (1) by the client to send a PANIC message to ZLight replicas, (2) by the replicas to generate and send a signed message containing their history, (3) by the client to invoke Backup with the abort/init history, and (4) by the (next) client to get the abort history from Backup and initialize the next ZLight instance. Note that we ensure that for each aborted request, the history contains $r$ requests. We reproduced each experiment three times and observed a variance of less than $3 \%$.

Figure 5 shows the switching time (in milliseconds) as a function of the history size when the number of tolerated faults equals 1. As described in Section 4.2.4, ZLight uses a checkpointing mechanism triggered every 128 requests. Moreover, to account for requests that can be received while a replica is performing a checkpoint, we assume that the history size can grow up to 250 requests. Note that in our current implementation, the history size is actually bounded: when the history is full, requests are blocked until a checkpoint is completed. We plot two different curves: one corresponds to the case when replicas do not miss any request. The other one corresponds to the case of missing requests, described in Section 4.4. More precisely, we assess the performance when 30\% 


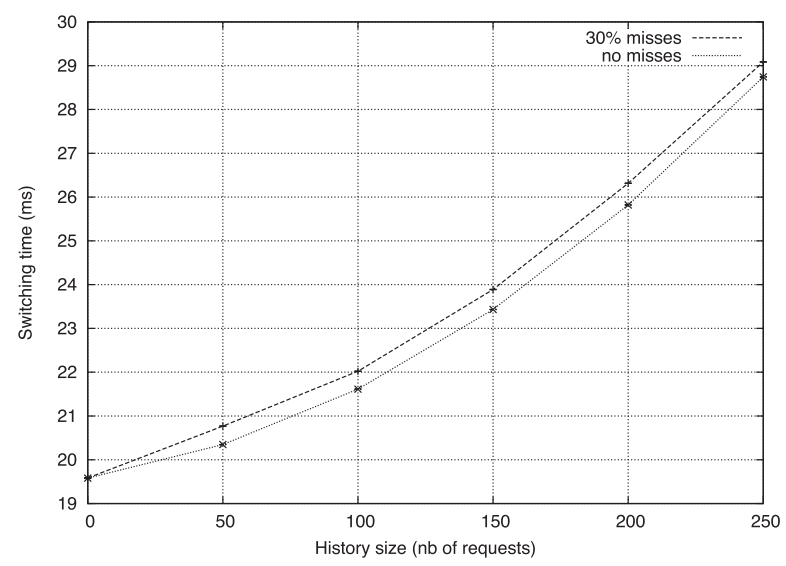

Fig. 5. Switching time as a function of the history size and the percentage of missing requests in replica histories.

of the requests are absent from the history of at least one replica upon receiving an init history containing signed message digests. Not surprisingly, we observe that the switching cost increases with the history size and that it is slightly higher in the case when replicas miss some requests (as replicas need to fetch the requests they miss). Interestingly, we see that the switching cost is low. It ranges between $19.7 \mathrm{~ms}$ and $29.2 \mathrm{~ms}$. This is very reasonable provided that faults are supposed to be rare in the environment for which Zyzzyva has been devised.

Furthermore, we observe that the switching cost grows faster than linearly. We argue that this is not an issue since the number of requests in histories is bounded by the checkpointing protocol. Finally, the switching cost could easily be higher in the case of a real application performing actual computations on requests that are reordered by the switching mechanism. However, it is important to notice that this extra cost would also be present in Zyzzyva, induced by the request replay during view changes.

\section{A NEW BFT PROTOCOL: ALIPH}

In this section, we demonstrate how we can build novel, very efficient BFT protocols using Abstract. We present a new protocol, called Aliph, that achieves up to $30 \%$ lower latency and up to $25 \%$ higher throughput than state-of-the-art protocols. The development of Aliph consisted of building two new instances of Abstract, each requiring less than 30\% of the code of state-of-the-art protocols, and reusing Backup (Section 4.3). In the following, we start with an overview of Aliph. We then present the two new Abstract instances it relies on. Finally, we assess its performance.

\subsection{Protocol Overview}

Aliph is a new BFT protocol that uses three Abstract implementations: Backup, Quorum, and Chain. The Backup protocol has been introduced in Section 4.3. A Quorum instance commits requests as long as there are no (1) server/link failures, (2) client Byzantine failures, or (3) contention. Quorum implements a very simple communication pattern (one round trip of message exchange) that is very efficient when there is no contention. The Chain protocol provides exactly the same progress guarantees as ZLight (Section 4.2); that is, it commits requests as long as there are no server/link failures or Byzantine clients. Chain implements a pipeline pattern that is very efficient under contention, unlike Quorum. Aliph uses the following static switching ordering to orchestrate its underlying protocols: Quorum-Chain-Backup-Quorum-Chain-Backup.... In other words, Quorum is initially active. As soon as it aborts (e.g., due to 
Table I. Characteristics of State-of-the-Art BFT Protocols

\begin{tabular}{|l|c|c|c|c|c|}
\cline { 2 - 6 } \multicolumn{1}{c|}{} & PBFT & Q/U & HQ & Zyzzyva & Aliph \\
\hline Number of replicas & $\mathbf{3 f + 1}$ & $5 f+1$ & $\mathbf{3 f + 1}$ & $\mathbf{3 f + 1}$ & $\mathbf{3 f \mathbf { + 1 }}$ \\
\hline Number of MAC operations at the bottleneck replica & $2+\frac{8 f}{b}$ & $2+4 \mathrm{f}$ & $2+4 \mathrm{f}$ & $2+\frac{3 f}{b}$ & $\mathbf{1 +} \frac{2 f+1}{b}$ \\
\hline Number of 1-way messages in the critical path & 4 & $\mathbf{2}$ & 4 & 3 & $\mathbf{2}$ \\
\hline
\end{tabular}

Note: Bold entries denote protocols with the lowest known cost.

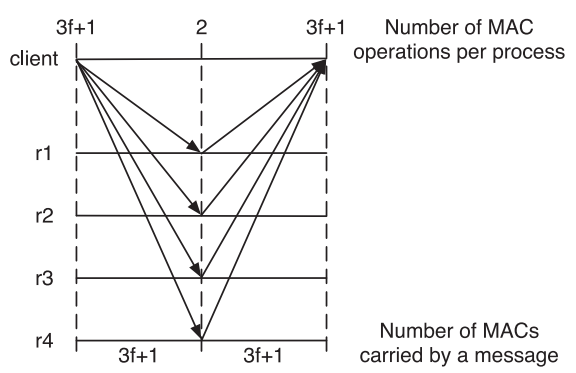

Fig. 6. Communication pattern of Quorum.

contention), it switches to Chain. Chain commits requests until it aborts (e.g., due to asynchrony). Aliph then switches to Backup, which commits $k$ requests (see Section 4.3). When Backup commits $k$ requests, it aborts, switches back to Quorum, and so on.

The characteristics of Aliph are summarized in Table I, considering the metrics of Kotla et al. [2010]. As we can see, Aliph achieves better throughput and latency than existing protocols and is optimally resilient. More precisely, Aliph is the first optimally resilient protocol that achieves a latency of 2 one-way message delays when there is no contention. It is also the first protocol for which the number of MAC operations at the bottleneck replica tends to 1 (under high contention when batching of messages is enabled): $50 \%$ less than required by state-of-the-art protocols.

In the next two sections, we describe Quorum and Chain. We focus on the common case as both Quorum and Chain use the same panicking mechanism and checkpointing protocol as ZLight that we presented in Sections 4.2.2 and 4.2.4, respectively.

\subsection{Quorum}

In this section, we present Quorum, an Abstract implementation used in the Aliph protocol to guarantee low latency when there is no contention. We first describe the Quorum protocol. We then give its pseudo-code.

5.2.1. Protocol Description. The communication pattern implemented in Quorum is depicted in Figure 6. This pattern is very simple: it requires only one round trip of message exchange between a client and replicas to commit a request. Namely, the client sends the request to all replicas that speculatively execute it and send a reply to the client. As in ZLight, replies sent by replicas contain a digest of their history. The client checks that the histories sent by the $3 f+1$ replicas match. If that is not the case, or if the client does not receive $3 f+1$ replies, the client invokes a panicking mechanism. This is the same as in ZLight (Section 4.2.2): (1) the client sends a PANIC message to replicas, (2) replicas stop executing requests on reception of a PANIC message, and (3) replicas send back a signed message containing their history. The client collects $2 f+1$ signed messages containing replica histories and generates an abort history. Note that, unlike ZLight, Quorum does not tolerate contention: concurrent requests can be executed in different orders by different replicas, inducing the current Quorum instance to abort. This is not the case in ZLight, as requests are ordered by the primary. 


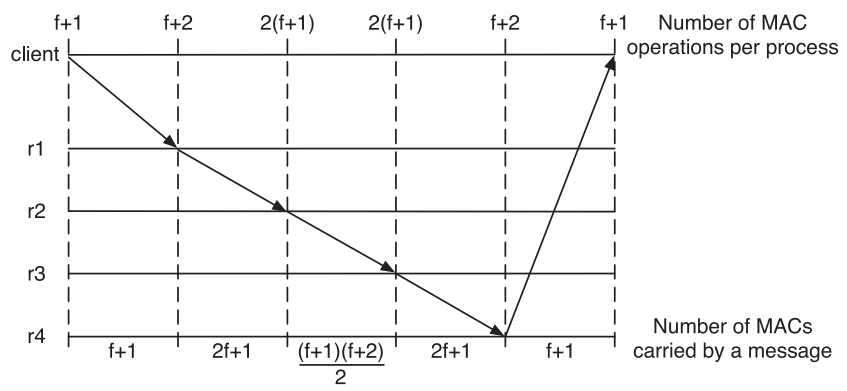

Fig. 7. Communication pattern of Chain.

Quorum makes Aliph the first BFT protocol to achieve a latency of 2 one-way message delays while only requiring $3 f+1$ replicas (Q/U [Abd-El-Malek et al. 2005] has the same latency but requires $5 f+1$ replicas). Given its simplicity and efficiency, it might seem surprising not to have seen it published earlier. We believe that Abstract made that possible because we could focus on the weaker (and hence easier to implement) Abstract specification, without having to consider the numerous difficult corner cases that occur outside the "common case."

Finally, let us note that the implementation of Quorum is very simple. It requires only 3,200 lines of $\mathrm{C} / \mathrm{C}++$ code (including the code for panicking and checkpointing that is the same as the respective code used in the ZLight and Chain protocols).

5.2.2. Pseudo-Code. To follow we give the pseudo-code of Quorum using the same notations as the ones presented in Figure 4. Moreover, as for ZLight, we omit to mention, for the sake of brevity, that upon receiving a message $m$, a process $p$ first checks that $m$ has a valid authenticator. We do not describe the pseudo-code of the panicking and checkpointing mechanisms as they are shared between Quorum and ZLight. Finally, we maintain the definitions of a replica logging and executing a request from ZLight (Section 4.2).

Step Q1. On $\operatorname{Invoke}_{i}(r e q)$, client $c$ sends message $\langle\text { REQ, req, } i\rangle_{\mu_{c, \Sigma}}$ to all replicas and triggers timer $T$ set to $2 \Delta$.

Step Q2. On receiving $\langle\mathrm{REQ}, \text { req, } i\rangle_{\mu_{c . \Sigma}}$ from client $c$, if req.t. is higher than $t_{j}[c]$, then replica $r_{j}$ (i) updates $t_{j}[c]$ to req.t. $t_{c}$, (ii) logs and executes $r e q$, and (iii) sends $\left\langle\mathrm{RESP}, \text { reply }_{j}, D\left(\mathrm{LH}_{j}\right), i, \text { req. } t_{c}, r_{j}\right\rangle_{\mu_{r_{j}, c}}$ to $c$.

Step Q3. Identical to Step Z4 of ZLight.

\subsection{Chain}

In this section, we present Chain, an Abstract implementation used in the Aliph protocol to ensure high throughput under contention. The Chain protocol shares similarities with the protocol presented in van Renesse and Schneider [2004]. Nevertheless, there is a significant difference between the two protocols: the protocol presented in this article tolerates Byzantine faults, whereas the protocol presented in van Renesse and Schneider [2004] only tolerates crash faults, which makes it significantly simpler. This section is organized as follows: we first describe the Chain protocol, and we then give its pseudo-code.

5.3.1. Protocol Description. The communication pattern implemented in Chain is presented in Figure 7. As we see, Chain organizes replicas in a pipeline. All replicas know the fixed ordering of replica IDs (called chain order); the first (resp., last) replica is called the head (resp., the tail). Without loss of generality, we assume an ascending ordering by replica IDs, where the head (resp., tail) is replica $r_{1}$ (resp., $r_{3 f+1}$ ). 
In Chain, a client invokes a request by sending it to the head, who assigns sequence numbers to requests. Then, each replica $r_{i}$ forwards the message to its successor $\overrightarrow{r_{i}}$, where $\overrightarrow{r_{i}}=r_{i+1}$. The exception is the tail whose successor is the client: upon receiving the message, the tail sends the reply to the client. Similarly, replica $r_{i}$ in Chain accepts a message only if sent by its predecessor $\overleftarrow{r_{i}}$, where $\overleftarrow{r_{i}}=r_{i-1}$; the exception is the head, which accepts requests only from the client.

Chain tolerates Byzantine failures by ensuring (1) that the content of a message is not modified by a Byzantine replica before being forwarded, (2) that no replica in the chain is bypassed, and (3) that the reply sent by the tail is correct. To provide those guarantees, Chain relies on a novel authentication method we call chain authenticators $(\mathrm{CAs})$. CAs are lightweight MAC authenticators, requiring processes to generate (at most) $f+1$ MACs (in contrast to $3 f+1$ in traditional authenticators). CAs guarantee that if a client commits request req, every correct replica logs req (i.e., appends it to its local history). CAs, along with the inherent throughput advantages of a pipeline pattern, are key to Chain's dramatic throughput improvements over other BFT protocols. We describe later how CAs are used in Chain.

Replicas and clients generate CAs in order to authenticate the messages they send. Each CA contains MACs for a set of processes called successor set. The successor set of clients consists of the $f+1$ first replicas in the chain order. The successor set of a replica $r_{i}$ depends on its position $i$ : (1) for the first $2 f$ replicas, the successor set comprises the next $f+1$ replicas in the chain, whereas (2) for other replicas $(i>2 f)$, the successor set comprises all subsequent replicas in the chain, as well as the client. Dually, when process $p$ receives a message $m$ it verifies $m$; that is, it checks whether $m$ contains a correct MAC from the processes from $p$ 's predecessor set (a set of processes $q$ such that $p$ is in $q$ 's successor set). For instance, replica $r_{2}$ verifies that the message contains a valid MAC from the replica $r_{1}$ (i.e., the head) and the client, whereas the client verifies that the reply it gets contains a valid MAC from the last $f+1$ replicas in the chain order.

Whereas all Chain replicas log a request by appending it to their local history, only $f+1$ last replicas execute requests and calculate the application-level reply. The reply is sent to the client only by the tail. To make sure that the reply sent by the tail is correct, the $f$ processes that precede the tail in the chain order append a digest of the reply to the message. ${ }^{11}$

When the client receives a correct reply, it commits it. On the other hand, when the reply is not correct, or when the client does not receive any reply (e.g., due to a Byzantine replica that discards the request), the client sends a PANIC message to all replicas. Just like in ZLight and Quorum, when replicas receive a PANIC message, they stop executing requests and send back a signed message containing their history. The client collects $2 f+1$ signed messages containing replica histories and generates an abort history.

Chain makes Aliph the first protocol in which the number of MAC operations at the bottleneck replica tends to 1 . Indeed, the bottleneck replica in Chain is the $f+1$-st replica. This replica needs to (1) read a MAC from the client, (2) read a MAC from its $f$ predecessors in the chain, and (3) write a MAC for its $f+1$ first successors in the chain. Replicas in the chain can forward multiple requests in a single batch and can generate a single MAC for the batch of requests. The first $f+1$ replicas do nevertheless need to read the MAC written by the client. Consequently, the bottleneck replica (i.e., the

\footnotetext{
${ }^{11}$ In some cases, sequential request execution by $f+1$ Chain replicas might impact latency of Chain for large values of $f$. On the other hand, (1) values of $f$ are typically small, and (2) there are practical workloads with processing times much lower than the communication latency, for example, when a metadata service is replicated.
} 
$f+1$-st replica) will perform $1+\frac{2 f+1}{b}$ MAC operations per request, with $b$ being the number of requests per batch. State-of-the-art protocols [Kotla et al. 2010; Castro and Liskov 2002] do all require at least two MAC operations at the bottleneck server (with the same assumption on batching). The reason that this number tends to 1 in Chain can intuitively be explained by the fact that these are two distinct sets of replicas that read a MAC from the client (the $f+1$ first replicas in the chain) and write a MAC to the client (the $f+1$ last replicas in the chain). In contrast, state-of-the-art protocols require some replicas to both read a MAC from the clients and write a MAC to the clients.

We have implemented Chain in $\mathrm{C} / \mathrm{C}++$. The implementation requires about 4,300 lines of code (including the panicking and checkpointing code). This is about $30 \%$ of the code size of state-of-the-art protocols.

5.3.2. Pseudo-Code. To follow we describe the pseudo-code of Chain. We use the same notations as for ZLight and Quorum. These notations are summarized in Figure 4. Moreover, we assume that every CHAIN message sent by a process $p$ contains the CA generated by $p$, as well as the MACs $p$ received from its predecessor $\overleftarrow{p}$ and that are destined to processes in $p$ 's successor set. Finally, we do not describe the pseudo-code of the panicking and checkpointing mechanisms that are shared between Chain and ZLight.

Step C1. On $\operatorname{Invoke}_{i}($ req $)$, client $c$ sends the message $m^{\prime}=\langle$ CHAIN, req, $i\rangle$ to the head (say, $\left.r_{1}\right)$ and triggers the timer $T$ set to $(n+1) \Delta$.

Step C2. On receiving $m=\langle$ CHAIN, req, $i\rangle$ from client $c$, if (i) req. $t_{c}$ is higher than $t_{1}[c]$ and (ii) the head can verify client's MAC (otherwise the head discards $m$ ), then the head $r_{1}(\mathrm{i})$ updates $t_{1}[c]$ to req. $t_{c}$, (ii) increments $s n_{1}$, and (iii) sends $\left\langle\right.$ CHAIN, req, $\left.i, s n_{1}, \perp, \perp\right\rangle$ to $\overrightarrow{r_{1}}=r_{2}$.

Step C3. On receiving $m=\langle$ CHAIN, req $, i, s n, R E P L Y, L H D i g e s t\rangle$ from $\overleftarrow{r_{j}}$, if (i) it can verify MACs from all processes from its predecessor set against the content of $m$, (ii) $s n=s n_{j}+1$, and (iii) req. $t_{c}$ is higher than $t_{j}[c]$, then (i) replica $r_{j}$ updates $s n_{j}$ to $s n$ and $t_{j}[c]$ to req.t. $t_{c}$, (ii) if $r_{j}$ is one of the first $2 f$ replicas, it logs req, and otherwise $r_{j} \operatorname{logs}$ and executes req, and (iii) $r_{j}$ sends $\langle$ CHAIN, req, $i, s n, R E P L Y$, LHDigest $\rangle$ to $\overrightarrow{r_{j}}$, where $R E P L Y=L H D i g e s t=\perp$ in case of the first $2 f$ replicas, $R E P L Y=D\left(\right.$ reply $\left.y_{j}\right)$ and LHDigest $=D\left(L H_{j}\right)$ in case $j \in\{2 f+1 \ldots 3 f\}$, and $R E P L Y=$ reply $_{j}$ and LHDigest $=$ $D\left(L H_{j}\right)$ in case $r_{j}$ is tail. In case the MAC verification mentioned previously fails, replica stops executing Step C3 in instance $i$.

Step C4. If client $c$ receives 〈CHAIN, req, $i, *$, reply, LHDigest〉 from the tail before expiration of $T_{\text {chain }}$, and with MACs from the last $f+1$ replicas that authenticate req, $i$, LHDigest, and $D$ (reply) (or reply itself), then $c$ commits req with reply. Otherwise, the client triggers the panicking mechanism explained in Section 4.2.2 (Step P1).

\subsection{Performance Evaluation}

In this section, we evaluate the performance of Aliph. We ran all our experiments on a cluster of 17 identical machines, each equipped with a $1.66 \mathrm{GHz}$ biprocessor and 2GB of RAM. Machines run the Linux 2.6.18 kernel and are connected using a Gigabit Ethernet switch.

We first study the latency, throughput, and fault scalability using microbenchmarks [Castro and Liskov 2002; Kotla et al. 2010], varying the number of clients. In these microbenchmarks, clients invoke requests in closed loop; that is, a client does not invoke a new request before it gets a reply for a previous one. ${ }^{12}$ The benchmarks are denoted

\footnotetext{
${ }^{12}$ Although closed-loop microbenchmarks are not always representative of the behavior of real systems [Schroeder et al. 2006], we use these microbenchmarks to enable fair comparison with previously reported results (e.g., [Castro and Liskov 2002; Kotla et al. 2010; Clement et al. 2009]).
} 
Table II. Latency Improvement of Aliph for the 0/0, 4/0, and 0/4 Benchmarks, Without Contention

\begin{tabular}{|l|c|c|c||c|c|c||c|c|c|}
\cline { 2 - 10 } \multicolumn{1}{c|}{} & \multicolumn{3}{c|}{$0 / 0$ Benchmark } & \multicolumn{3}{c||}{$4 / 0$ Benchmark } & \multicolumn{3}{c|}{$0 / 4$ Benchmark } \\
\cline { 2 - 10 } \multicolumn{1}{c|}{} & $\mathrm{f}=1$ & $\mathrm{f}=2$ & $\mathrm{f}=3$ & $\mathrm{f}=1$ & $\mathrm{f}=2$ & $\mathrm{f}=3$ & $\mathrm{f}=1$ & $\mathrm{f}=2$ & $\mathrm{f}=3$ \\
\hline Q/U & $8 \%$ & $14,9 \%$ & $33,1 \%$ & $6,5 \%$ & $13,6 \%$ & $22,3 \%$ & $4,7 \%$ & $20,2 \%$ & $26 \%$ \\
\hline Zyzzyva & $31,6 \%$ & $31,2 \%$ & $34,5 \%$ & $27,7 \%$ & $26,7 \%$ & $15,6 \%$ & $24,3 \%$ & $26 \%$ & $15,6 \%$ \\
\hline PBFT & $49,1 \%$ & $48,8 \%$ & $44,5 \%$ & $36,6 \%$ & $38,4 \%$ & $26 \%$ & $37,6 \%$ & $38,2 \%$ & $29 \%$ \\
\hline
\end{tabular}

$x / y$, where $x$ is the request payload size (in kilobytes) and $y$ is the reply payload size (in kilobytes). We then proceed by studying the performance of Aliph under faults. Finally, we perform an experiment in which the input load dynamically varies.

We evaluate PBFT and Zyzzyva because the former is considered the "baseline" for practical BFT implementations, whereas the latter is considered state of the art. Moreover, Zyzzyva systematically outperforms HQ [Kotla et al. 2010]; hence, we do not evaluate HQ. Finally, we benchmark Q/U as it is known to provide better latency than Zyzzyva under certain conditions. Note that Q/U requires $5 f+1$ servers, whereas other protocols we benchmark only require $3 f+1$ servers.

PBFT and Zyzzyva implement two optimizations: request batching by the primary, and client multicast (in which clients send requests directly to all the servers and the primary only sends ordering messages). All measurements of PBFT are performed with batching enabled as it always improves performance. This is not the case in Zyzzyva. Therefore, we assess Zyzzyva with or without batching depending on the experiment. As for the client multicast optimization, we show results for both configurations every time we observe an interesting behavior.

Aliph also implements two optimizations. First, the progress property of Chain is slightly different from the one described earlier in the article: Chain aborts requests as soon as replicas detect that there is no contention (i.e., there is only one active client since at least $2 s$ ). This avoids executing Chain when there is no contention. Second, Chain replicas add information in their abort history to specify that they aborted because of the lack of contention. We modified Backup so that in such case, it only commits one request and aborts. Consequently, when there is no contention, Aliph switches to Quorum, which is very efficient in such a case.

Finally, let us note that the PBFT code base underlies both Zyzzyva and Aliph, which ensures a fair comparison between these three protocols. To also ensure that the comparison with $\mathrm{Q} / \mathrm{U}$ is fair, we evaluate a simple best-case implementation that uses the same code base and that is described in Kotla et al. [2010]. Consequently, all protocols are implemented in $\mathrm{C}++$, rely on MD5 for computing message digests, and use UMAC as MAC type. Finally, regarding networking primitives, all protocols use UDP and IP Multicast, as described in the respective papers. The only exception is Chain, which uses TCP. As our experiments demonstrate, the pipeline pattern of Chain coupled with the use of TCP gives Chain a significant advantage over other protocols under high load.

5.4.1. Latency. We first assess the latency in a system without contention, with a single client issuing requests. The improvement of Aliph over Q/U, PBFT, and Zyzzyva is reported in Table II for all microbenchmarks $(0 / 0,0 / 4$, and 4/0) and for a maximal number of server failures $f$ ranging from 1 to 3 . We observe that Aliph consistently outperforms other protocols. The reason that the latency achieved by Aliph is very low is that it uses Quorum when there is no contention. These results confirm the theoretical analysis (see Table I, Section 5.1). The results show that Q/U also achieves a good latency with $f=1$. This is not surprising provided that $\mathrm{Q} / \mathrm{U}$ and Quorum use the same communication pattern. Nevertheless, when $f$ increases, the performance of $\mathrm{Q} / \mathrm{U}$ decreases significantly. The reason is that $\mathrm{Q} / \mathrm{U}$ requires $5 f+1$ replicas and 


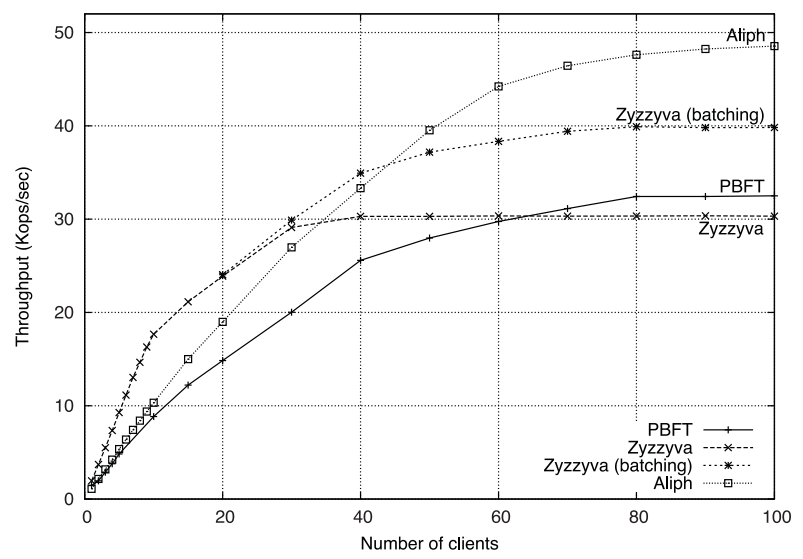

Fig. 8. Throughput for the $0 / 0$ benchmark $(f=1)$.

both clients and servers perform additional MAC computations compared to Quorum. Moreover, the significant improvement of Aliph over Zyzzyva (31\% at $f=1)$ can be easily explained by the fact that Zyzzyva requires 3 one-way message delays in the common case, whereas Aliph (Quorum) only requires 2 one-way message delays.

5.4.2. Throughput. In this section, we present throughput results obtained running the $0 / 0,0 / 4$, and $4 / 0$ microbenchmarks under contention. We do not report results for $\mathrm{Q} / \mathrm{U}$ since it is known to perform poorly under contention. Notice that in all the experiments presented in this section, Chain is active in Aliph. The reason is that, due to contention, there is always a point when a request sent to Quorum reaches replicas in a different order, which results in a switch to Chain. As there are no failures in the experiments presented in this section, Aliph never switches to Backup. Consequently, Chain commits all the subsequent requests.

The results presented in this section show that Aliph consistently and significantly outperforms other protocols, starting from a certain number of clients that depends on the benchmark. Below this threshold, Zyzzyva achieves higher throughput than other protocols.

0/0 benchmark. Figure 8 plots the throughput achieved with the $0 / 0$ benchmark by the various protocols when $f=1$. We ran Zyzzyva with and without batching. For PBFT, we present only the results with batching, since they are substantially better than those obtained without batching. We observe that Zyzzyva with batching performs better than PBFT, which itself achieves higher peak throughput than Zyzzyva without batching (this is consistent with the results of Kotla et al. [2010] and Singh et al. [2008]).

Moreover, Figure 8 shows that with up to 40 clients, Zyzzyva achieves the best throughput. With more than 40 clients, Aliph starts to outperform Zyzzyva. The peak throughput achieved by Aliph is $21 \%$ higher than that of Zyzzyva. The reason is that Aliph executes Chain, which uses a pipeline pattern to disseminate requests. This pipeline pattern brings two benefits: reduced number of MAC operations at the bottleneck server and better network usage: servers send/receive messages to/from a single server. Nevertheless, the Chain protocol is efficient only if its pipeline is fed-the link between any server and its successor in the chain is saturated. There are two ways to feed the pipeline: using large messages (see the next benchmark) or a large number of small messages (this is the case of the $0 / 0$ benchmark). Provided that in the microbenchmarks clients invoke requests in closed loop, it is necessary to have a large 


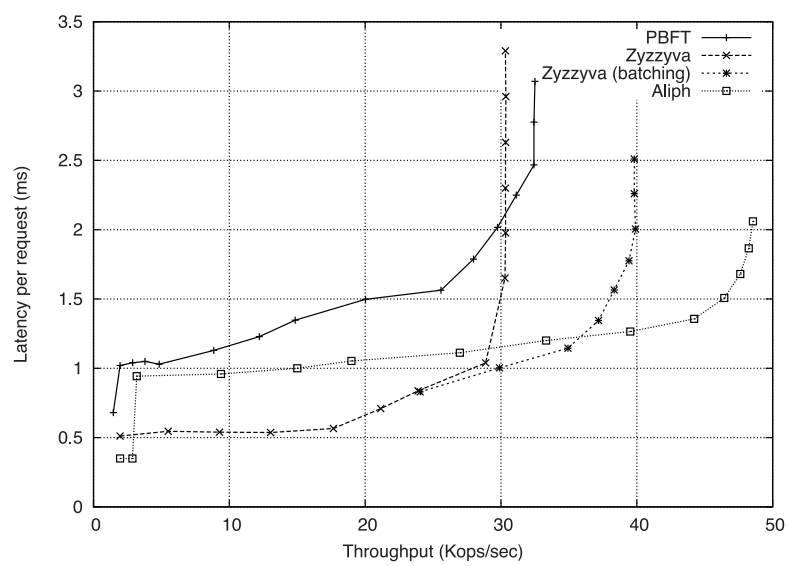

Fig. 9. Response time versus throughput for the $0 / 0$ benchmark $(f=1)$.

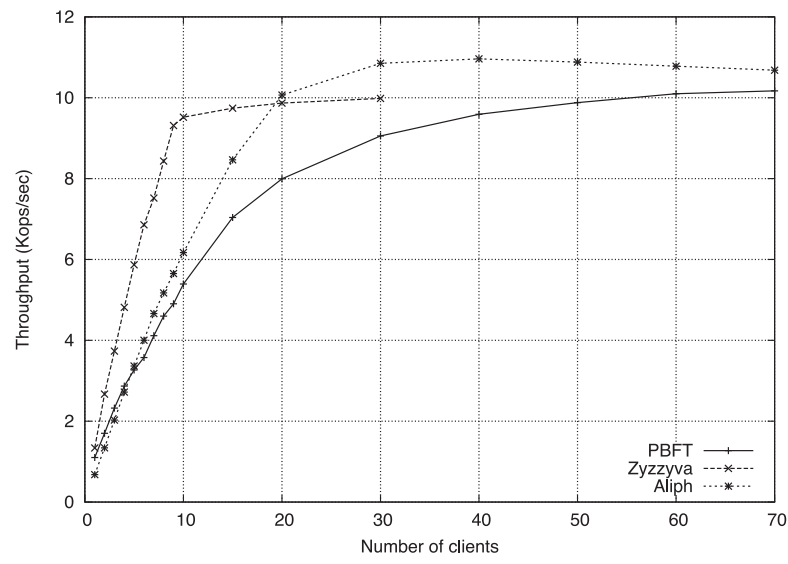

Fig. 10. Throughput for the $0 / 4$ benchmark $(f=1)$.

number of clients to issue a large number of requests. This explains why Aliph starts outperforming Zyzzyva only with more than 40 clients.

Figure 9 plots the response time of Zyzzyva (with and without batching), PBFT, and Aliph as a function of the achieved throughput. We observe that Aliph achieves consistently lower response time than PBFT. This stems from the fact that the message pattern implemented by PBFT is very complex, which increases the response time and limits the throughput of PBFT. Moreover, up to the throughput of 37Kops/sec, Aliph has a slightly higher response time than Zyzzyva. The reason is the pipeline pattern of Chain that results in a higher response time for low to medium throughput but stays nevertheless reasonable. Moreover, Aliph scales better than Zyzzyva: from 37Kops/sec, it achieves lower response time, since the messages are processed faster due to the higher throughput ensured by Chain. Hence, messages spend less time in waiting queues. Finally, we observe that for very low throughput, Aliph has a lower response time than Zyzzyva. This comes from the fact that Aliph uses Quorum when there is no contention, which significantly improves the response time of the protocol.

0/4 benchmark. Figure 10 shows the throughput of the various protocols for the $0 / 4$ microbenchmark when $f=1$. PBFT and Zyzzyva are using the client multicast 


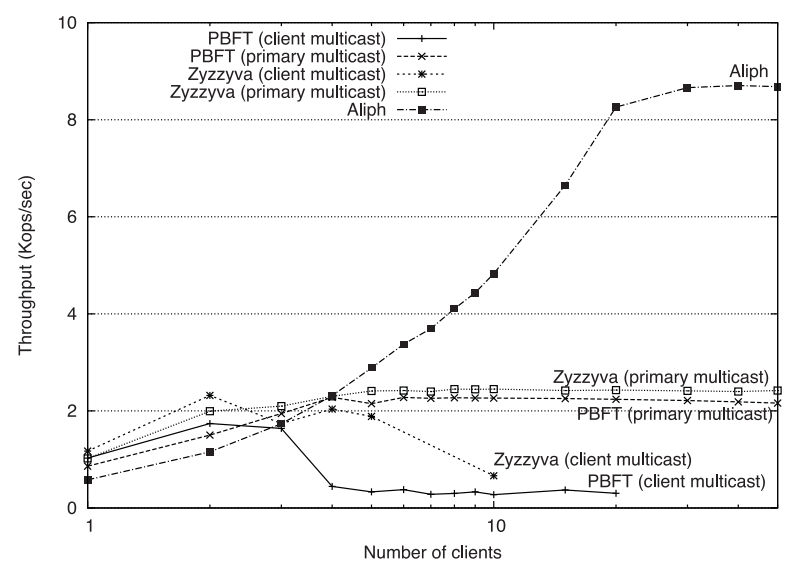

Fig. 11. Throughput for the $4 / 0$ benchmark $(f=1)$.

optimization. We observe that with up to 15 clients, Zyzzyva outperforms other protocols. Starting from 20 clients, Aliph outperforms PBFT and Zyzzyva. Nevertheless, the gain in peak throughput (7.7\% over PBFT and 9.8\% over Zyzzyva) is lower than the gain we observed with the $0 / 0$ microbenchmark. This can be explained by the fact that the dominating cost is now in sending replies to clients. This cost partly masks the cost of request ordering. In all protocols, there is only one server sending a full reply to the client (other servers send only a digest of the reply), which explains why the various protocols achieve a pretty close throughput.

4/0 benchmark. Figure 11 shows the results of Aliph, PBFT, and Zyzzyva for the $4 / 0$ microbenchmark with $f=1$. Notice the logarithmic scale for the x-axis, which we use to better highlight the behavior of the various protocols with small numbers of clients. For PBFT and Zyzzyva, we plot curves both with and without client multicast optimization. The graph shows that with up to three clients, Zyzzyva outperforms other protocols. With more than three clients, Aliph significantly outperforms other protocols. Its peak throughput is about $360 \%$ higher than that of Zyzzyva. The reason that Aliph is very efficient under high load and when requests are large was explained earlier in the context of the 0/0 benchmark. We attribute the poor performance of PBFT and Zyzzyva to the fact that when IP Multicast is used with large messages, this induces message losses that are inefficiently handled by the available prototypes. Moreover, we explain the performance drop observed for Zyzzyva and PBFT when the client multicast optimization is used (Figure 11) by the fact that enabling this optimization increases the number of message losses (due to a higher number of message collisions).

5.4.3. Impact of the Request Size. In this experiment, we study how protocols are impacted by the size of requests. Figure 12 shows the peak throughput of Aliph, PBFT, and Zyzzyva as a function of the request size for $f=1$ (the response size is kept at $0 \mathrm{k}$ ). To obtain the peak throughput of PBFT and Zyzzyva, we benchmarked both protocols with and without the client multicast optimization and with different batching sizes for Zyzzyva. Interestingly, the behavior we observe is similar to that observed using simulations in Singh et al. [2008]: the performance gap between PBFT and Zyzzyva diminishes with the increase in payload. Indeed, starting from 128B payloads, both protocols have almost identical performance. Figure 12 also shows that Aliph sustains high peak throughput with all message sizes, which is again the consequence of Chain being active under contention. 


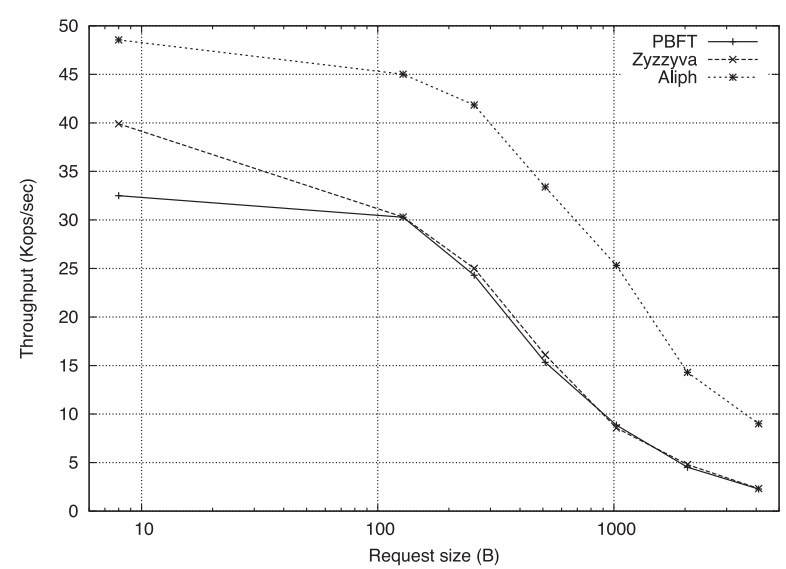

Fig. 12. Peak throughput as a function of the request size $(\mathrm{f}=1)$.

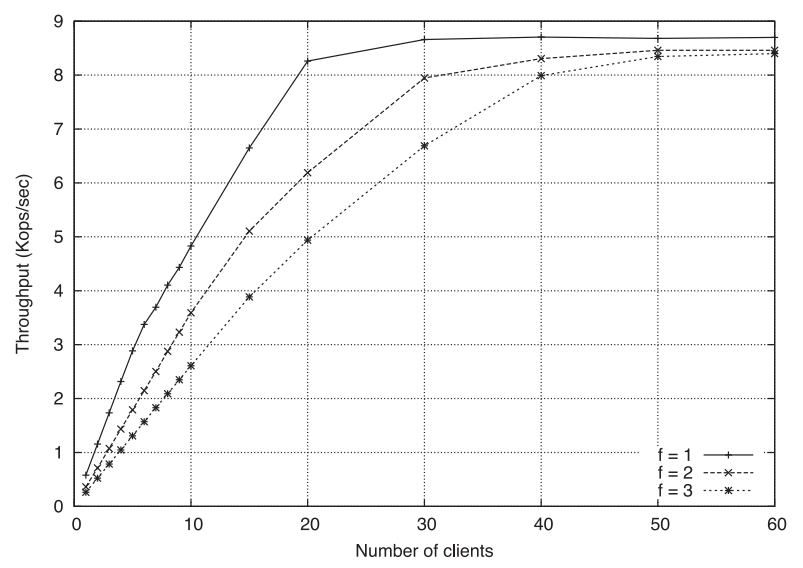

Fig. 13. Impact of the number of tolerated failures $f$ on the Aliph throughput.

5.4.4. Fault Scalability. One important characteristic of BFT protocols is their behavior when the number of tolerated server failures $f$ increases. Figure 13 depicts the performance of Aliph for the 4/0 benchmark when $f$ varies between 1 and 3 . We do not present results for PBFT and Zyzzyva as their peak throughput is known to suffer only a slight impact [Kotla et al. 2010]. Figure 13 shows that this is also the case for Aliph. The peak throughput at $f=3$ is only $3.5 \%$ lower than that achieved at $f=1$. We also observe that the number of clients that Aliph requires to reach its peak throughput increases with $f$. This can be explained by the fact that Aliph uses Chain under contention. The length of the pipeline used in Chain increases with $f$. Hence, more clients are needed to feed the Chain and reach the peak throughput.

5.4.5. Behavior of Aliph in Case of Faults. In this section, we assess the behavior of Aliph when one replica crashes. The experiment proceeds as follows. We consider four replicas $(f=1)$ and one client that issues 15,000 requests. After the client sends 2,000 requests, we crash one replica, which recovers 10s later. Consequently, during 10 s, only three replicas are active. We compare two strategies: in the first strategy, when Aliph switches to Backup, Backup always commits $k=1$ request. In the second strategy, when Aliph switches to Backup, it commits $k=2^{i}$, where $i$ is the number of invocations of Backup 

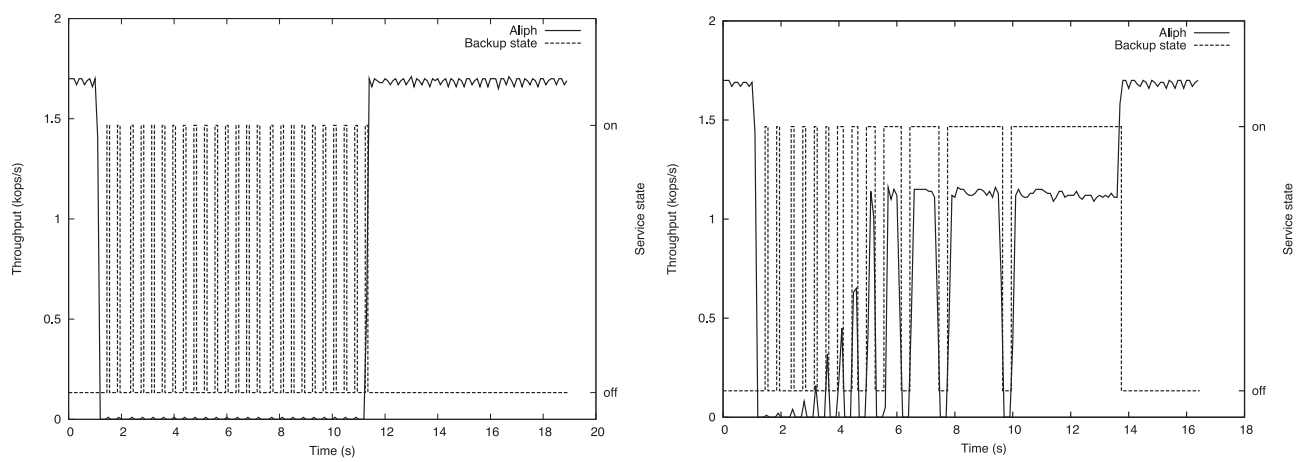

(a) Behavior under faults, when Aliph switches to (b) Behavior under faults, when Aliph switches to Backup for one request.

Backup for $2^{i}$ requests.

Fig. 14. Behavior of Aliph in case of faults.

since the beginning of the experiment. Note that in its current version, Aliph combines both strategies by exponentially increasing $k$ while maintaining the exponential curve initially very flat for reasons discussed in Section 4.3.

The behavior of Aliph with the first strategy is depicted in Figure 14(a). When only three replicas are active, Quorum and Chain cannot commit requests and Aliph switches to Backup for every single request. We depict on the y-axis both the throughput achieved by Aliph and the periods during which Backup is active. Not surprisingly, the throughput of Aliph is very low in this case.

Figure 14(b) shows the behavior of Aliph with the second strategy. We observe that the throughput is significantly higher because Backup is used to process an exponentially increasing number of requests. We can also observe that, although the four replicas are active at time $t=11 s$, Aliph switches back to Quorum only around time $t=14 \mathrm{~s}$. This is due to the fact that Backup had to process 8,192 requests before Aliph could switch. We point out that if the replica is down for a long time, Aliph will end up executing Backup for a very large number of requests. This means that, during a very long time period, the performance of Aliph will be that of Backup. We therefore periodically reset the number $k$ of requests that Backup processes before aborting.

5.4.6. Dynamic Workload. Finally, we study the performance of Aliph under a dynamic workload (i.e., fluctuating contention). We compare its performance to that achieved by Zyzzyva and by Chain alone. We do not present results for Quorum alone as it does not perform well under contention. The experiments consist of having 30 clients issuing requests of different sizes, namely, $0 \mathrm{k}, 0.5 \mathrm{k}, 1 \mathrm{k}, 2 \mathrm{k}$, and $4 \mathrm{k}$, with response size kept at $0 \mathrm{k}$. Clients do not send requests all at the same time: the experiment starts with a single client issuing requests. Then we progressively increase the number of clients until it reaches 10 . We then simulate a load spike with 30 clients simultaneously sending requests. Finally, the number of clients decreases, until there is only one client remaining in the system.

Figure 15 shows the performance of Aliph, Zyzzyva, and Chain. For each protocol, clients were invoking the same number of requests. Moreover, requests were invoked after the preceding clients had completed their bursts. First, we observe that Aliph is the most efficient protocol: it completes the experiment in $42 s$, followed by Zyzzyva (68.1s) and Chain (77.2s). Up to time $t=15.8 s$, Aliph uses Quorum, which performs much better than Zyzzyva and Chain. Starting at $t=15.8$, contention becomes too high for Quorum, which switches to Chain. At time $t=31.8 \mathrm{~s}$, there is only one client in the system. After $2 s$ spent with only one client in the system, Chain in Aliph starts 


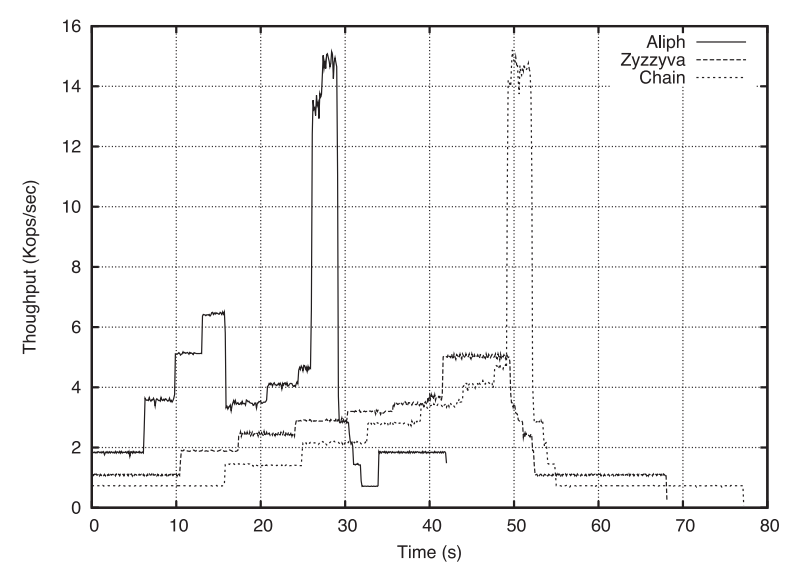

Fig. 15. Throughput under dynamic workload.

aborting requests due to the low load optimization described earlier. Consequently, Aliph switches to Backup and then to Quorum. This explains the increase in throughput observed at time $t=33.8 \mathrm{~s}$. We also observe on the graph that Chain and Aliph are more efficient than Zyzzyva when there is a load spike: they achieve a peak throughput about three times higher than that of Zyzzyva. On the other hand, Chain and Aliph have slightly lower performance than Zyzzyva under medium load (i.e., from $16 s$ to $26 s$ on the Aliph curve). This suggests an interesting BFT protocol that would combine Quorum, Zyzzyva, Chain, and Backup.

\section{MAKING ALIPH ROBUST: $R$-ALIPH}

The Aliph protocol presented in Section 5 achieves excellent performance in the "common" case, that is, when the network is synchronous and when both clients and replicas are benign. Unfortunately, as we show in this section, a Byzantine client or a Byzantine replica can attack this protocol and drastically reduce its performance. This fragility of BFT protocols is well known and motivated the development of three so-called robust protocols: Spinning [Veronese et al. 2009], Prime [Amir et al. 2011], and Aardvark [Clement et al. 2009]. In this section, we first study the performance achieved by Aliph when clients or replicas attack the protocol (Section 6.1). We then briefly describe existing robust protocols and compare their performance under the same attacks (Section 6.2). We then describe R-Aliph, a robust version of the Aliph protocol (Section 6.3). Finally, we evaluate the performance of $R$-Aliph both with and without attacks (Section 6.4).

\subsection{Aliph Under Attack}

We study the performance of Aliph under four different, representative attacks inspired from those used in Clement et al. [2009]:

-Client flooding: in this attack, one of the clients is Byzantine: it implements a brute force denial of service attack by repeatedly sending $9 \mathrm{kB}$ messages to the replicas.

-Malformed client requests: in this attack, one of the clients is Byzantine: it sends requests with an invalid authenticator that can only be authenticated by a subset of the replicas (including the primary in Backup and the head in Chain).

- Processing delay: in this attack, one Byzantine replica (the primary in Backup (PBFT), the head in Chain, and a randomly chosen replica in Quorum) delays the ordering of requests it receives from clients by $10 \mathrm{~ms}$. 
Table III. Peak Throughput (req/s) of Aliph Both in the Absence of Attacks and Under Various Attacks (0/0 Microbenchmark)

\begin{tabular}{|c|c|c|c|c|c|}
\cline { 2 - 6 } \multicolumn{1}{c|}{} & $\begin{array}{c}\text { Without } \\
\text { Attack }\end{array}$ & $\begin{array}{c}\text { Client } \\
\text { Flooding }\end{array}$ & $\begin{array}{c}\text { Malformed } \\
\text { Requests }\end{array}$ & $\begin{array}{c}\text { Processing } \\
\text { Delay }(10 \mathrm{~ms})\end{array}$ & $\begin{array}{c}\text { Replica } \\
\text { Flooding }\end{array}$ \\
\hline Aliph & 55,575 & $30,733(-44,7 \%)$ & $0(-100 \%)$ & $2,629(-95,3 \%)$ & $0(-100 \%)$ \\
\hline
\end{tabular}

-Replica flooding: in this attack, one of the replicas (different from the primary in Backup and the head in Chain) is Byzantine: it fails to process protocol messages and implements a brute force denial of service attack by repeatedly sending $9 \mathrm{kB}$ messages to other replicas.

The performance results for the 0/0 microbenchmark are reported in Table III (we observed similar behavior with the $4 / 0$ and 0/4 microbenchmarks). ${ }^{13}$

We observe that the throughput of Aliph drops to 0 when malformed requests are sent or when one replica floods other replicas. This is explained by the fact that in both cases, Quorum and Chain are not able to commit requests, which induces a switching to Backup. Backup relies on PBFT, which is unable to sustain a nonnull throughput in these cases as already observed in Clement et al. [2009].

Regarding the "processing delay" attack, the throughput of Aliph drops to about 2,629 requests per second. This result is explained by the fact that requests are invoked in a closed-loop manner; that is, a client does not invoke a new request before it gets a reply for a previous one. Consequently, delaying the ordering of requests decreases the throughput achieved by the protocol. Moreover, the overall latency perceived by the clients is below the threshold beyond which they timeout, panic, and trigger a switching to Backup. Consequently, the throughput remains at this very low value.

Finally, regarding the "client flooding" attack, the throughput of Aliph decreases, but less than with the other attacks. This is explained as follows. When running under this attack, Quorum is not able to commit requests, which induces a switch to Chain. The latter relies on TCP, and, consequently, correct clients can issue requests at a high throughput despite the fact that a malicious client is trying to flood the network.

\subsection{A Brief Overview of "Robust" BFT Protocols}

The fact that efficient BFT protocols are often fragile (i.e., perform poorly under attack) motivated the development of so-called robust BFT protocols: Spinning [Veronese et al. 2009], Prime [Amir et al. 2011], and Aardvark [Clement et al. 2009]. These protocols aim at achieving good performance when the network is synchronous, despite the presence of Byzantine faulty clients and replicas. To follow, we briefly describe these three protocols.

Spinning [Veronese et al. 2009] is a robust BFT protocol based on PBFT [Castro and Liskov 2002]. The idea underlying Spinning is to perform regular primary changes after each (fixed-size) batch of requests in order to limit the impact a Byzantine primary can have. Moreover, to further limit the impact of Byzantine primaries, Spinning uses a blacklisting mechanism that works as follows. Requests are sent to all replicas and, as soon as a nonprimary replica receives a request, it starts a timer $\left(S_{\text {timeout }}\right)$ and waits for a request ordering message from the primary for this request. In case of a timeout, the

\footnotetext{
${ }^{13}$ Notice that in order to accommodate the networking requirements of protocols that are studied later in this section, these experiments have been performed on another experimental testbed made of eightcore machines. Each machine has ten $1 \mathrm{~GB}$ network cards and runs Linux 2.6.35. This explains the slight performance difference with results reported in previous sections.
} 
Table IV. Peak Throughput (req/s) of Spinning, Prime, and Aardvark Both in the Absence of Attacks and Under Various Attacks (0/0 Microbenchmark)

\begin{tabular}{|l|c|c|c||c|c|}
\cline { 2 - 6 } \multicolumn{1}{c|}{} & $\begin{array}{c}\text { Without } \\
\text { Attack }\end{array}$ & $\begin{array}{c}\text { Client } \\
\text { Flooding }\end{array}$ & $\begin{array}{c}\text { Malformed } \\
\text { Requests }\end{array}$ & $\begin{array}{c}\text { Processing } \\
\text { Delay (10ms) }\end{array}$ & $\begin{array}{c}\text { Replica } \\
\text { Flooding }\end{array}$ \\
\hline Spinning & 37,116 & $19,164(-48.4 \%)$ & $36,986(-0.3 \%)$ & $18,529(-50.1 \%)$ & $21,975(-40.8 \%)$ \\
\hline Prime & 6,682 & $1,445(-78.4 \%)$ & $6,596(-1.3 \%)$ & $3,648(-45.4 \%)$ & $0(-100 \%)$ \\
\hline Aardvark & 31,510 & $30,280(-3.9 \%)$ & $31,336(-0.1 \%)$ & $25,997(-17.5 \%)$ & $28,599(-9.2 \%)$ \\
\hline
\end{tabular}

current primary is blacklisted (i.e., it will no longer become a primary in the future ${ }^{14}$ ) and $S_{\text {timeout }}$ is doubled.

Another robust protocol that has been designed is Prime [Amir et al. 2011]. In Prime, clients can send their requests to any replica in the system. Replicas periodically exchange the requests they receive from clients. Consequently, replicas are aware of the set of requests that should be ordered (i.e., for which they expect ordering messages from the primary). Moreover, even when there are no requests to order, the primary must periodically send (empty) ordering messages. That way, nonprimary replicas expect to receive ordering messages at a given frequency. To improve the accuracy of the expected frequency, replicas monitor the network performance. Specifically, replicas periodically measure the round-trip time between each pair of them. This measure allows replicas to compute the maximum delay that should elapse between two consecutive sending of ordering messages by a correct primary. If the primary becomes slower than what is expected by the replicas, then it is replaced.

Finally, a third robust protocol that has been designed is Aardvark [Clement et al. 2009], also based on PBFT. Aardvark implements a number of mechanisms to ensure good performance despite the presence of Byzantine clients and replicas. First, the protocol limits the degradation that Byzantine clients can cause by isolating their traffic and by implementing a smart authentication mechanism that limits the impact of malformed client requests. Second, the protocol limits the impact that a faulty replica can have by isolating the traffic induced by the different replicas: namely, each replica uses a distinct NIC to communicate with every other replica. Third, the protocol limits the damage that a faulty primary may cause. To this end, all replicas monitor the throughput at which the primary is ordering requests. During the first $5 \mathrm{~s}$ of the primary being active, the primary is expected to achieve a throughput at least equal to $90 \%$ of the maximum throughput achieved by the primary replicas of the $n$ preceding views (where $n$ is the number of replicas). The expected throughput is then periodically raised by a factor of 0.01 . As soon as a primary does not meet the throughput expectations, a primary change occurs.

In Table IV, we report the performance of Spinning, Prime, and Aardvark for the 0/0 microbenchmark, both in the absence of attacks and under the three attacks described in the previous section. ${ }^{15}$ We do use a slightly modified "malformed request" attack because the three studied protocols use signatures to authenticate messages rather than MAC authenticators. Hence, in this section, a malformed request is a request with an invalid signature.

We can make several observations. First, in the absence of attacks, robust protocols are much less efficient than fast BFT protocols, namely, ZLight and Chain. For instance, the throughput of Chain is $76 \%$ higher than that of Aardvark. This performance difference between fast and robust protocols is expected and well known [Clement et al.

\footnotetext{
${ }^{14}$ If $f$ replicas are already blacklisted, then the oldest one is removed from the blacklist to ensure the liveness of the system.

${ }^{15}$ We use the original code bases for the three protocols. Nevertheless, we improved the networking stack of the Spinning protocol and enabled batching in order to increase its performance.
} 
2009]: it is due to the performance overhead induced by the various mechanisms that robust protocols implement to limit the impact of Byzantine clients and replicas. Second, we observe that, overall, the performance of robust protocols is less impacted by attacks than that of Aliph. Third, we observe that there are significant differences among robust protocols. Aardvark consistently achieves better performance under attack than both Spinning and Prime. The reason is that Aardvark combines accurate monitoring of the primary progress and network isolation between pairs of replicas, whereas Spinning and Prime only monitor the primary progress.

\subsection{R-Aliph, a Robust Version of Aliph}

The conclusion we can draw from the two previous sections is that distributed system designers seemingly have the choice between either (1) a BFT protocol (e.g., Aliph) that achieves very good performance when there are no attacks, but that achieves very bad or no throughput under attacks, or (2) a BFT protocol (e.g., Aardvark) that achieves a lower throughput when there are no attacks (about $43 \%$ less efficient than Aliph in the 0/0 microbenchmark), but that is only slightly impacted by attacks.

In this section, we show how, using Abstract, we designed $R$-Aliph, a protocol that almost achieves the best of both worlds: $R$-Aliph is almost as efficient as Aliph when there are no attacks, and as efficient as Aardvark when there are attacks. To achieve this goal, we built $R$-Aliph following four main principles:

- (Principle P1) Backup is implemented on top of Aardvark (and is thus resilient to attacks).

- (Principle P2) Quorum and Chain are only executed if they sustain a better throughput than the one Backup (i.e., Aardvark) would sustain.

-(Principle P3) Quorum and Chain are only executed if they are fair with respect to different clients.

- (Principle P4) The time needed to switch among the three protocols is not impacted by the presence of Byzantine clients and Byzantine replicas.

It is trivial to enforce Principle P1. We describe next how the remaining three principles are enforced.

In order to make sure that $R$-Aliph executes Quorum and Chain only if they sustain a better throughput than the one Backup would sustain (see Principle P2), replicas executing Quorum or Chain periodically monitor the throughput achieved by the protocol and check that it is higher than an expected throughput. If a replica detects that this is not the case, it becomes unhappy, stops processing requests, and triggers a protocol switching (we explain this later on, under Principle P4). In the following, we explain how replicas in $R$-Aliph (a) set their throughput expectations and (b) monitor the current throughput:

(a) In setting throughput expectation thresholds, $R$-Aliph replicas leverage the throughput expectations that are computed inside Backup (i.e., Aardvark) when it is executed. More specifically, when R-Aliph switches to Quorum or Chain, the throughput that each replica $r_{i}$ expects is the maximum over all throughput expectations $r_{i}$ computed when executing Backup (i.e., Aardvark).

(b) When executing Quorum or Chain (just like with Zyzzyva/ZLight), a replica cannot accurately compute the throughput based on what it observes locally, that is, neither based on the requests it orders nor based on the checkpoint messages it receives from other replicas. For instance, a Byzantine replica in Quorum (or Zyzzyva/ZLight) can send a checkpoint message pretending that it executed a request but postpone the sending of the reply to that request, thus reducing the overall throughput without being caught by the client (e.g., if the client receives 
the reply before the timer expires, as in the "processing delay" attack described in Section 6.1). Similarly, the tail replica in Chain can send a checkpoint message pretending that it replied to a client but postpone the sending of the reply to the client.

Consequently, the only way for replicas to accurately monitor the throughput in $R$-Aliph is to ask clients to send to replicas feedback messages confirming they committed the requests they previously sent. ${ }^{16}$ In $R$-Aliph, Quorum and Chain replicas calculate the throughput using feedback messages every 128 committed requests with a replica taking feedback into account only for a request it previously executed. In our prototype, to limit the overhead of feedback messages, clients only send them every five requests they commit. Moreover, in the case of Quorum, feedback messages are piggybacked to "common case" requests within REQ messages. In addition, if a replica does not perform another throughput calculation in the time Backup would have committed 128 requests (see also point (a)), a replica immediately becomes unhappy and stops processing further requests.

In order to make sure that $R$-Aliph executes Quorum and Chain only in case clients are treated fairly (Principle P3), the replicas implement the following mechanism (inspired by Aardvark): replicas track client requests: they check that, after having received a request req, no single client issues feedback for two requests received after req (which might be a sign that one of the replicas is unfair and delays req). If a replica detects that this is not the case, it stops processing requests and triggers a protocol switching (see Principle P4 implementation later for details on switching). Observe that replicas in Quorum receive all requests directly from clients and can thus accurately track requests. This is not the case for Chain: the head can forward requests in an arbitrary order, thus preventing accurate tracking. The only way to make sure that replicas accurately track requests is to have clients send feedback messages to indicate they issued some requests. To limit the overhead of these messages in Chain, they are piggybacked to feedback messages containing information about committed requests.

Finally, in order to ensure that the time needed to switch between the three protocols is not impacted by Byzantine clients and/or Byzantine replicas (Principle P4), R-Aliph relies on three main ideas:

(a) First, each replica bounds the number of uncheckpointed requests it adds to its local history. This in turn bounds the amount of state that might need to be transferred during the switching. In our implementation, this bound is set to 384 requests, which is not limiting performance (i.e., setting this bound to a higher value does not increase the peak throughput achieved by $R$-Aliph).

(b) Second, $R$-Aliph leverages the isolation of networking and processing resources that Aardvark (that $R$-Aliph uses in Backup) anyway requires. More precisely, as illustrated in Figure 16, (i) each replica uses a dedicated NIC to communicate with clients-note that this particular NIC is also used in Chain to exchange common-case protocol messages among replicas, (ii) each replica uses a dedicated set of NICs to communicate with other replicas, (iii) each replica picks messages on the set of NICs dedicated to other replicas in a round-robin manner, and (iv) each replica disables a NIC dedicated to communicating with another replica if the latter sends invalid messages or many more messages than the other replicas on average. This combination of mechanisms ensures a robust implementation of point-to-point channels between each pair of replicas: that is, even if a Byzantine

\footnotetext{
${ }^{16}$ Notice here that some BFT protocols can rely on replicas only to monitor throughput—this is the case with protocols that have an explicit commit phase among replicas, as in PBFT and Aardvark.
} 


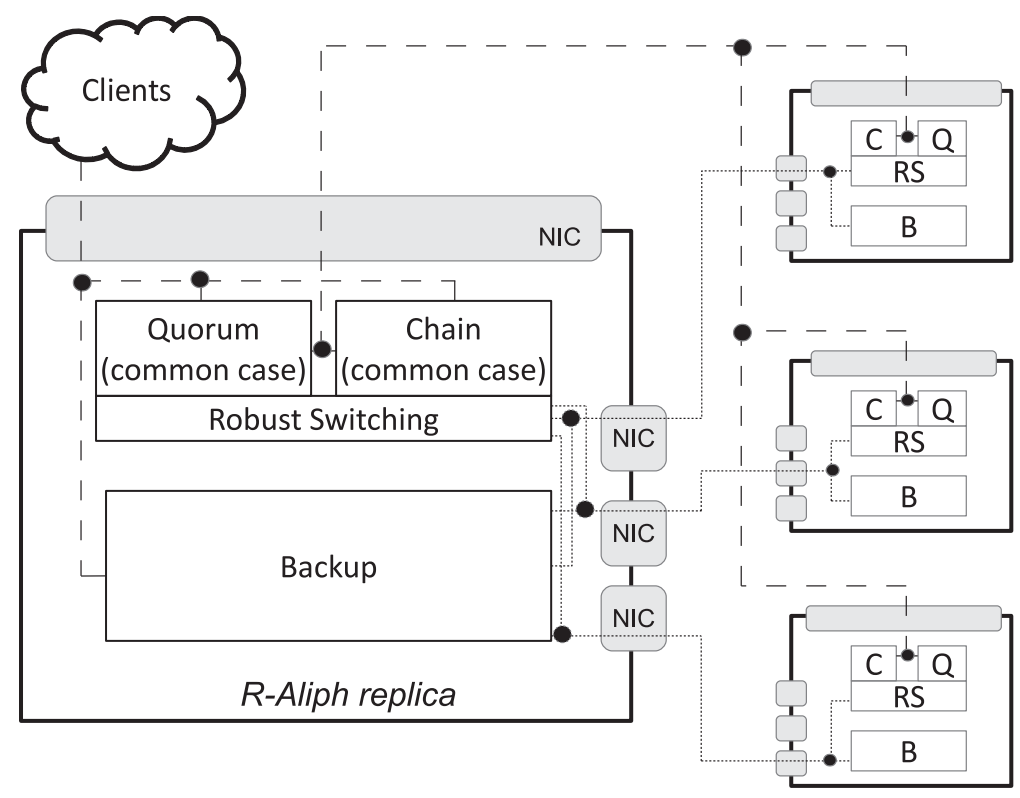

Fig. 16. Architecture of $R$-Aliph replicas.

client or a Byzantine replica floods the network, this will not prevent any pair of correct replicas from communicating efficiently.

(c) Third, clients are not involved in the switching protocol (although they can panic if they want) - otherwise, since a replica is connected to all clients using a single NIC, a Byzantine client could perform a denial-of-service attack and arbitrarily delay the protocol switching. This is achieved as follows. A replica wishing to trigger protocol switching (because it detects that Quorum or Chain either do not sustain adequate throughput or are unfair, or if some client panics) acts itself as a client: it invokes a noop request on the current Abstract (i.e., Quorum or Chain) and immediately panics (without waiting for the timer in Steps Q1/C1). The replica then completes Step P3 of the panicking/aborting subprotocol and switches, acting as a client, to the next Abstract instance. Then, since (i) the size of local histories is limited, (ii) clients are not involved in the aborting protocol (beyond the ability to panic), and (iii) each pair of replicas is connected by a dedicated point-to-point channel, we are guaranteed that Byzantine clients and/or Byzantine replicas cannot impact the upper bound on time required to perform a protocol switching.

\subsection{Evaluation}

In this section, we evaluate $R$-Aliph. We first assess its overhead with respect to Aliph. We then study its behavior under attacks. Finally, we assess the worst-case switching time under attack.

6.4.1. Overhead of R-Aliph. $R$-Aliph has an overhead that is mainly caused by feedback messages sent by clients to notify replicas of the Chain protocol that they sent and committed requests. To assess this overhead, we run an experiment without attacks and compare the throughput of $R$-Aliph to that achieved by Aliph. We vary the request size from $0 \mathrm{kB}$ to $10 \mathrm{kB}$ and we use null replies (we obtained comparable results with nonnull reply sizes). Results are reported in Figure 17. We can make two observations. First, we observe that the maximum throughput decrease is below 6\%. This is 


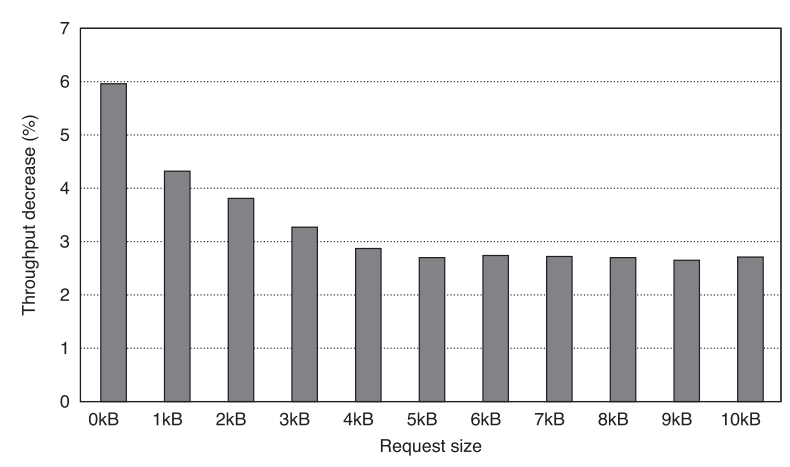

Fig. 17. Throughput decrease of $R$-Aliph with respect to Aliph for various request sizes and null replies.

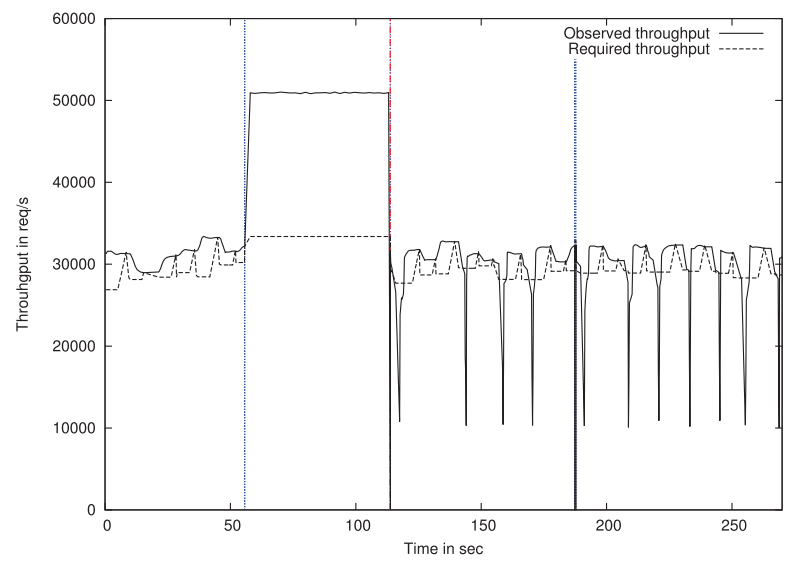

Fig. 18. Behavior of $R$-Aliph under a "processing delay" attack.

very reasonable: in "common case," $R$-Aliph is still $65 \%$ more efficient than Aardvark. Second, we observe that the overhead of $R$-Aliph decreases when the size of requests increases. For instance, with $4 \mathrm{kB}$ requests, the throughput decrease of $R$-Aliph with respect to Aliph is below 3\%. The reason that the overhead decreases when increasing the request size is that the relative size of feedback client messages becomes lower.

6.4.2. Behavior of R-Aliph Under Attack. We assessed the performance of $R$-Aliph under the attacks described in Section 6.1. Figure 18 presents the behavior of $R$-Aliph during the "processing delay" attack. We have observed similar behaviors for other attacks. Therefore, to avoid redundancy, we do not provide figures for other attacks. On the $\mathrm{x}$-axis, we report the time (in seconds). On the y-axis, we report both the throughput that was expected by replicas (dashed line) and the throughput that $R$-Aliph actually sustained (solid line). The dotted vertical lines represent protocol switchings. The experiment is as follows. One hundred clients inject $8 \mathrm{~B}$ requests in a closed-loop manner and receive 8B replies during all of the experiment. Initially, $R$-Aliph executes Backup, configured to execute a fixed amount of requests. There is no ongoing attack yet and Backup sustains a throughput of about $31,500 \mathrm{req} / \mathrm{s}$, which is in line with what we previously reported in Table IV. Then, at time $55 \mathrm{~s}, R$-Aliph switches to Quorum. As there is contention, Quorum is not able to commit requests and immediately aborts. Subsequently, $R$-Aliph switches to Chain. The latter sustains a much higher throughput than Backup (and thus a much higher throughput than that expected by replicas). 
Table V. Worst-Case Switching Time (in ms) of R-Aliph Both in the Absence of Attacks and Under Various Attacks

\begin{tabular}{|l|c|c|c|c|}
\hline $\begin{array}{l}\text { Without } \\
\text { Attack }\end{array}$ & $\begin{array}{c}\text { Client } \\
\text { Flooding }\end{array}$ & $\begin{array}{c}\text { Replica Is } \\
\text { Unfair }\end{array}$ & $\begin{array}{c}\text { Processing } \\
\text { Delay (10ms) }\end{array}$ & $\begin{array}{c}\text { Replica } \\
\text { Flooding }\end{array}$ \\
\hline 60.36 & 62.49 & 60.52 & 63.92 & 63.24 \\
\hline
\end{tabular}

At time 114s, the attack starts. The replica acting as head in Chain adds a $10 \mathrm{~ms}$ processing delay to all messages it receives. We have profiled the system and observed that a correct replica in the Chain starts noticing that Chain is not behaving properly and triggers a switching about $7 \mathrm{~ms}$ after the attack started. Again, system profiling shows that it takes about $63 \mathrm{~ms}$ for Chain to switch to Backup. The latter executes requests despite the attack. Its throughput is slightly impacted ( $-21 \%$ on average). Indeed, we can observe periodic performance drops that are explained by the fact that the Aardvark protocol used in Backup regularly changes the primary replica and one-fourth of the time, the elected primary is the Byzantine replica that adds a $10 \mathrm{~ms}$ processing delay. This induces a short performance drop before a new primary is elected. At time 187 s, after Backup executed a fixed amount of requests, $R$-Aliph switches to Quorum. The latter is not able to commit requests (because of contention). $R$-Aliph subsequently switches to Chain that does not sustain the required throughput (because of the attack). Profiling reveals that it again takes about $5 \mathrm{~ms}$ for a correct replica in the Chain to notice that Chain is slow and about $20 \mathrm{~ms}$ to switch to Backup. Note that switching at around $187 \mathrm{~s}$ is faster compared to the switching at around $114 \mathrm{~s}$ when the attack was initially launched. Profiling reveals that this is because the history of replicas contains a much smaller amount of requests.

6.4.3. Worst-Case Switching Time. In this section, we assess the worst-case switching time both without attacks and under the different attacks described in Section 6.1. The time we measure corresponds to the elapsed time between the creation of the first panicking message and the time when the replicas have switched to the next protocol. To assess the worst-case switching time, we perform several switchings in a loop, as explained in Section 4.6, with half of the replicas having a full request history (384 ten-kilobyte requests) and half of the replicas having an empty request history. This configuration induces the largest possible state transfer among replicas. Results are reported in Table V. We observe that the worst-case switching time is low and that it is only marginally impacted in the presence of attacks. This is easily explained by the fact that (1) the processing and networking resources needed by the switching mechanism are isolated, and (2) clients are not involved in the switching protocol. Therefore, correct replicas can perform switching without being impacted by the presence of Byzantine replicas and clients.

\section{RELATED WORK}

The idea of aborting if "something goes wrong" is old. It underlies, for instance, the seminal two-phase commit protocol [Gray 1978]: abort can be decided if there is a failure or some database server votes "no." The idea was also explored in the context of mutual exclusion: a process in the entry section can abort if it cannot enter the critical section [Jayanti 2003]. Abortable consensus was proposed in Chen [2007] and Boichat et al. [2003]. In the first case, a process can abort if a majority of processes cannot be reached, whereas in the second case, a process can abort if there is contention. The latter idea was generalized for arbitrary shared objects in Attiya et al. [2005] and then Aguilera et al. [2007]. In Aguilera et al. [2007], a process can abort and then query the object to seek whether the last query of the process was performed. This query can, however, abort if there is contention. Our notion of abortable state machine replication 
is different. First, the condition under which Abstract can abort is a generic parameter: it can express, for instance, contention, synchrony, or failures. Second, in case of abort, Abstract returns (without any further query) what is needed for recovery in a Byzantine context; namely, an unforgeable history. This, in turn, can be used to invoke another, possibly stronger Abstract. This ability is key to the composability of Abstract instances.

Several examples of speculative protocols, distinguishing an optimistic phase from a recovery one, were discussed in the survey of Pedone [2001]. These speculation ideas were used in the context of Byzantine state machine replication, for example, in HQ [Cowling et al. 2006] and Zyzzyva [Kotla et al. 2010]. We are, however, the first to clearly separate the phases and encapsulate them within first-class, well-specified modules that can each be designed, tested, and proved independently. In a sense, Abstract enables the construction of a BFT protocol as the composition of as many (gracefully degrading) phases as desired, each with a "standard" interface. This allows for an unprecedented flexibility in BFT protocol design that we illustrated with Aliph, a BFT protocol that combines three different phases. Similarly, with $R$-Aliph, we illustrated how one can quickly devise and implement a BFT protocol ensuring good performance despite attacks. While we described Aliph and R-Aliph and showed that, albeit simple, they outperform existing BFT protocols, Aliph and R-Aliph are simply the starting point for Abstract.

To maintain the assumption of a threshold $f$ of replica failures, BFT systems need to ensure failure independence [Gashi et al. 2007; Garcia et al. 2011]. An established technique used in ensuring failure independence is n-version programming, which mandates a different BFT implementation for each replica, with the goal of reducing the probability of identical software faults across replicas. While Abstract does not alleviate the need for n-version programming, this may be less costly and more feasible due to the inherently reduced code sizes and complexities involved with Abstract implementations. In addition, abstractions like BASE [Castro et al. 2003], which enable reuse of off-theshelf service implementations, can be used complementarily to our approach.

Since the publication of the preliminary, conference version of this work [Guerraoui et al. 2010], several papers that exploit Abstract-like reconfiguration have been published. In particular, CheapBFT [Kapitza et al. 2012] implements Abstract-like reconfiguration with switching through replicas assuming an FPGA-based trusted subsystem to reduce the resource overhead of BFT. In the common case, CheapBFT relies on a novel, optimistic protocol that uses $f+1$ replicas, whereas in the case of failures, CheapBFT falls back to a protocol called MinBFT [Veronese et al. 2013] (itself also based on a trusted subsystem) that uses $2 f+1$ replicas. It is precisely for such usages demonstrated by CheapBFT, which diverge from the classical BFT model we assume in this article, that we defined Abstract as a specification with properties that reason about clients' histories rather than about the state and the number of replicas that implement Abstract. Indeed, Abstract specification deliberately does not reason about the number of replicas, their local state or state relative to other replicas, or the fault model. By adopting such an approach, we do not restrict the use of Abstract to the classical BFT model, but also allow for the use of Abstract in the trusted BFT model [Kapitza et al. 2012], vanilla crash-failure model, or any other failure model.

\section{CONCLUSION AND FUTURE WORK}

Byzantine fault-tolerant state machine replication (BFT) protocols are notoriously difficult to design, implement, and prove correct. In this article, we presented Abstract, a framework for the design and reconfiguration of abortable replicated state machines. Using Abstract, we incrementally developed new BFT protocols with a fraction of the complexity required to develop full-fledged BFT protocols. We build BFT protocols as 
sequences of Abstract instances, each designed, implemented, and proved independently. Such protocols are not only simpler to design but also efficient and robust.

In this article, we have implemented several BFT protocols (AZyzzyva, Aliph, and $R$-Aliph) that consist of lightweight Abstract implementations (ZLight, Quorum, and Chain) designed to be efficient in the "common case," typically when the system is synchronous and there are no replica faults. In all protocols, we reused existing BFT protocols such as PBFT [Castro and Liskov 2002] and Aardvark [Clement et al. 2009] to handle the faulty case by wrapping them into a powerful Abstract instance called Backup.

In future work, several directions can be interesting to explore, for example, using the concepts that underly Abstract in the context of Byzantine-resilient storage [Hendricks et al. 2007] or devising signature-free switching. Moreover, we believe that an interesting research challenge lies in devising effective heuristics for dynamic switching among Abstract instances. While we described Aliph and R-Aliph and showed that, albeit simple, they outperform existing BFT protocols, Aliph and R-Aliph are simply the starting point for Abstract. The idea of dynamic switching depending on the system conditions seems very promising; such a scheme could monitor the current system conditions and implement smart heuristics to switch to the seemingly most appropriate Abstract instance.

\section{APPENDIX. CORRECTNESS PROOFS}

In this appendix, we give the correctness proofs of ZLight (Section 4.2), Quorum (Section 5.2), and Chain (Section 5.3). We omit the correctness proof of Backup (Section 4.3), which is straightforward due to the properties of the underlying BFT protocol. Finally, the proofs of liveness of our compositions trivially rely on the assumption of an exponentially increasing Backup configuration parameter $k$ (see Section 4.3).

Since ZLight and Quorum share many similarities, we give their correctness proof together. This is followed by the proof of Chain.

\section{A.1. ZLight and Quorum}

In this section, we prove that ZLight and Quorum implements Abstract. We first prove the common properties of the two implementations and then focus on the only different property (Progress).

Well-formed commit indications. It is easy to see that the reply returned by a commit indication for a request req always equals $r e p\left(h_{r e q}\right)$, where (commit history) $h_{r e q}$ is a uniquely defined sequence of requests. Indeed, by Step Z4/Q3, in order to commit a request, a client needs to receive identical digests (LHDigest) of some history $h^{\prime}$ and identical reply digests from all $3 f+1$ replicas, including from at least $2 f+1$ correct replicas. By Step Z3 of ZLight (resp., Step Q2 of Quorum), a digest of the reply sent by a correct replica is $D\left(r e p\left(h^{\prime}\right)\right)$. Hence, $h^{\prime}$ is exactly the commit history $h_{r e q}$ and is uniquely defined due to our assumption of collision-free digests.

Moreover, since a correct replica logs and then executes an invoked request req before sending a RESP message in Step Z4 (resp., Q3), it is straightforward to see that if req is committed with a commit history $h_{\text {req }}$, then req is in $h_{\text {req }}$.

Validity. For any request req to appear in a commit or abort history, at least $f+1$ replicas must have sent a history (or a digest of a history) containing req to the client (see Step Z4/Q3 for commit histories and Step P3 for abort histories). Hence, at least one correct replica appended req to its local history. By Step Z3/Q2, the correct replica $r_{j}$ appends $r e q$ to its local history only if $r_{j}$ receives a REQ message with a valid MAC from a client. This MAC is, in turn, present only if some client invoked req, or if req is contained in some verified (valid) init history. 
Moreover, by Step Z3/Q2, no replica logs/executes the same request twice (since every replica maintains $t_{j}[c]$ ). Hence, no request appears twice in any local history of a correct process, and consequently, no request appears twice in any commit history. In the case of abort histories, no request appears twice by construction (see Step P3, Section 4.2.2).

Termination. By assumption of a quorum of $2 f+1$ correct replicas and fair-loss links: since correct clients (resp., replicas) periodically retransmit the PANIC (resp., ABORT) messages (Step P1): (1) correct replicas eventually receive the PANIC message sent by correct client $c$ and (2) $c$ eventually receives $2 f+1$ ABORT messages from correct replicas (sent in Step P2). Hence, if correct client $c$ panics, it eventually aborts invoked request req, in case $c$ does not commit req beforehand.

To prove Commit and Abort Ordering, we first prove the following Lemma.

Lemma A.1. Let $r_{j}$ be a correct replica and $\mathrm{LH}_{j}^{\text {req }}$ the state of $\mathrm{LH}_{j}$ upon $r_{j}$ logs req. Then, $L H_{j}^{r e q}$ remains a prefix of $\mathrm{LH}_{j}$ forever.

Proof. A correct replica $r_{j}$ modifies its local history $L H_{j}$ only in Step Z3/Q2 by sequentially appending requests to $L H_{j}$. Hence, $L H_{j}^{r e q}$ remains a prefix of $L H_{j}$ forever.

Commit Order. Assume, by contradiction, that there are two different committed requests req (by benign client $c$ ) and req' (by benign client $c^{\prime}$ ), with different commit histories $h_{r e q}$ and $h_{r e q^{\prime}}$ such that neither is the prefix of the other. Since a benign client commits a request only if it receives in Step Z4/Q3 identical digests of replicas' local histories from all $3 f+1$ replicas, there must be a correct replica $r_{j}$ that sent $D\left(h_{\text {req }}\right)$ to $c$ and $D\left(h_{r e q^{\prime}}\right)$ to $c^{\prime}$ such that $h(r e q)$ is not a prefix of $h_{r e q^{\prime}}$ or vice versa-a contradiction with Lemma A.1.

Abort Order. First, we show that for every committed request req with the commit history $h_{r e q}$ and any ABORT message $m$ sent by a correct replica $r_{j}$ containing a (digest of a) local history $L H_{j}^{m}, h_{r e q}$ is a prefix of $L H_{j}^{m}$. Assume, by contradiction, that there are request $r e q^{\prime}$, correct replica $r_{j^{\prime}}$, and ABORT message $m^{\prime}$ such that the previous does not hold. Then, since a benign client needs to receive identical history digests from all replicas to commit a request (Step Z4/Q3), and since $r_{j^{\prime}}$ stops executing new requests before sending any ABORT message (Step P2), $r_{j^{\prime}}$ logged and executed req before sending $m^{\prime}$. However, by Lemma A.1, $h_{r e q^{\prime}}$ is a prefix of $L H_{j^{\prime}}^{m^{\prime}}$-a contradiction.

By Step P3, a client that aborts a request waits for $2 f+1$ ABORT messages including at least $f+1$ from correct replicas. Since any commit history $h_{r e q}$ is a prefix of every history sent in an ABORT message by any correct replica (as shown earlier), at least $f+1$ received histories will contain $h_{r e q}$ as a prefix, for any committed request req. Hence, by construction of abort histories (Step P3, Section 4.2.2), every commit history $h_{\text {req }}$ is a prefix of every abort history.

Init Order. By Step Z3+, Section 4.2.3, ${ }^{17}$ and Step P2, every correct replica must initialize its local history (with some valid init history) before sending any RESP or ABORT message. Since any common prefix $C P$ of all valid init histories is a prefix of any particular init history $I, C P$ is a prefix of every local history sent by a correct replica in an RESP or ABORT message. Init Order for commit histories immediately follows. In the case of abort histories, notice that out of $2 f+1$ ABORT messages received by a client on aborting a request in Step P3, at least $f+1$ are sent by correct replicas and contain local histories that have $C P$ as a prefix. Hence, by Step P3, $C P$ is a prefix of any abort history.

ZLight Progress. Recall that ZLight guarantees to commit clients' requests if there are no replica/link failures and Byzantine client failures. Recall also that, with no link

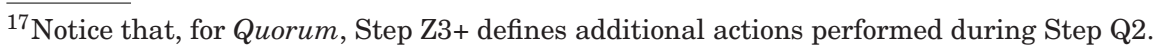


failures, message propagation time between two correct processes is bounded by $\Delta_{c}$ and that message processing time is bounded by $\Delta_{p}$. Also, in Step Z1, a client triggers a timer $T$ set to $3 \Delta$ (where $\Delta=\Delta_{c}+\Delta_{p}$ ). Then, to prove Progress, we prove a stronger property that no client executes Step P1 and panics (consequently, no client ever aborts and Progress follows from Termination).

Assume by contradiction that there is a client $c$ that panics and denote the first such time by $t_{P A N I C}$. Since no client is Byzantine, $c$ must be benign and $c$ invoked request $r e q$ at $t=t_{P A N I C}-3 \Delta$. Since no client panics by $t_{P A N I C}$, all replicas execute all requests they receive by $t_{P A N I C}$. Then, it is not difficult to see, since there are no link failures, that (1) by $t+\Delta_{c}$, the primary receives req, and by time $t+\Delta$, primary sends ORDER messages in Step Z2, and (2) by time $t+2 \Delta<t_{P A N I C}$, the replicas send RESP messages in Step Z3 for req. Since the primary is correct, all replicas execute all requests received before $t_{P A N I C}$ in the same order (established by the sequence numbers assigned by the primary). Hence, by $t+3 \Delta=t_{P A N I C}, c$ receives and processes $3 f+1$ identical replies (Step Z4), commits req, and never panics-a contradiction. if:

Quorum Progress. Recall that Quorum guarantees to commit clients' requests only

— there are no replica/link failures,

-no client is Byzantine, and

- there is no contention.

We assume that the timer $T$ triggered in Step Q1 is set to $2 \Delta$ (where $\Delta=\Delta_{c}+\Delta_{p}$ ). As in the proof of ZLight Progress, we prove a stronger property that no client executes Step P1 and panics.

Assume by contradiction that there is a client $c$ that panics and denote the first such time by $t_{P A N I C}$. Since no client is Byzantine, $c$ must be benign and $c$ invoked request $r e q$ at $t=t_{P A N I C}-2 \Delta$. Since no client panics by $t_{P A N I C}$, all replicas execute all requests they receive by $t_{P A N I C}$. Then, it is not difficult to see, since there are no link failures, that by time $t+\Delta<t_{P A N I C}$, all replicas receive req and send RESP message in Step Q2. Since there is no contention and all replicas are correct, all replicas order all requests in the same way and send identical histories to the clients. Hence, by $t+2 \Delta=t_{P A N I C}$, $c$ receives and processes $3 f+1$ identical replies (Step Q3), commits req, and never panics-a contradiction.

\section{A.2. Chain}

In this section, we prove that Chain implements Abstract with Progress equivalent to that of ZLight (see also Appendix A.1).

We denote the predecessor (resp., successor) set of the replica $r_{j}$ by $\overleftarrow{R_{j}}$ (resp., $\overrightarrow{R_{j}}$ ) We also denote by $\Sigma_{\text {last }}$ the set of the last $f+1$ replicas in the chain order, that is, $\Sigma_{\text {last }}=\left\{r_{j} \in \Sigma: j>2 t\right\}$. In addition, we say that correct replica $r_{j} \operatorname{logs}$ (resp., executes) req at position pos if $s n_{j}=$ pos when $r_{j}$ logs/executes req.

Before proving Abstract properties, we first prove two auxiliary lemmas. Notice also that Lemma A.1, Section A.1, extends to Chain as well.

Lemma A.2. If correct replica $r_{j}$ logs req (at position sn, at time $t_{1}$ ), then all correct replicas $s_{j}, 1 \leq j<i \log$ req (at position sn, before $t_{1}$ ).

Proof. By contradiction, assume the lemma does not hold and fix $r_{j}$ to be the first correct replica that logs req (at position $s n$ ) such that there is a correct replica $r_{x}(x<j)$ that never logs req (at position $s n$ ); we say $r_{j}$ is the first replica for which req skips. Since CHAIN messages are authenticated using CAs, $r_{j}$ logs $r e q$ at position $s n$ only if 
$r_{j}$ receives a CHAIN message with MACs authenticating req and $s n$ from all replicas from $\overleftarrow{R_{j}}$ authenticate req and $s n$, that is, only after all correct replicas from $\overleftarrow{R_{j}} \log r e q$ at position sn. If $r_{x} \in \overleftarrow{R_{j}}, r_{x}$ must have logged req at position $s n$-a contradiction. On the other hand, if $r_{x} \notin \overleftarrow{R_{j}}$, then $r_{j}$ is not the first replica for which req skips, since req skips for any correct replica (at least one) from $\overleftarrow{R_{j}}$ - a contradiction.

Lemma A.3. If benign client c commits req with history $h_{\text {req }}$ (at time $t_{1}$ ), then all correct replicas in $\Sigma_{\text {last }}$ execute req (before $t_{1}$ ) and the state of their local history upon executing req is $h_{\text {req. }}$.

Proof. To prove this lemma, notice that correct replica $r_{j} \in \Sigma_{\text {last }}$ generates a MAC for the client authenticating req and $D\left(h^{\prime}\right)$ for some history $h^{\prime}$ (Step C3) (1) only after $r_{j} \operatorname{logs}$ and executes req and (2) only if the state of $L H_{j}$ upon execution of req equals $h^{\prime}$. Moreover, by Step C3, no correct replica executes the same request twice. By Step C4, a benign client cannot commit req with $h_{r e q}$ unless it receives a MAC authenticating req and $D\left(h^{\prime}\right)$ from every correct replica in $\Sigma_{\text {last }}$-hence the lemma.

Well-formed commit indications. By Step C4, in order to commit a request req, a client needs to receive MACs authenticating LHDigest $=D\left(h^{\prime}\right)$ for some history $h^{\prime}$ and the reply digest from all replicas from $\Sigma_{\text {last }}$, including at least one correct replica. By Step C3, a digest of the reply sent by a correct replica is $D\left(r e p\left(h^{\prime}\right)\right)$. Hence, $h^{\prime}$ is exactly a commit history $h_{r e q}$ and is uniquely defined due to our assumption of collision-free digests.

Moreover, since a correct replica in $\Sigma_{\text {last }}$ logs and executes an invoked request before sending a CHAIN message in Step C3, it is straightforward to see that if req is committed with a commit history $h_{r e q}$, then $r e q$ is in $h_{r e q}$. Namely, notice that a client needs to receive the MAC for the same local history digest $D\left(h_{\text {req }}\right)$ from all $f+1$ replicas from $\Sigma_{\text {last }}$ including at least one correct replica $r_{j}$. By Step C3, $r_{j} \operatorname{logs} r e q$ and appends it to its local history $L H_{j}$ before authenticating the digest of $L H_{j}$; hence, req $\in h_{\text {req }}$.

Validity. For any request req to appear in an abort (resp., commit) history $h$, at least $f+1$ replicas must have sent $h$ (resp., a digest of $h$ ) in Step P2 (resp., Step C3) such that $r e q \in h$. Hence, at least one correct replica logged req.

Now, we show that correct replicas log only requests invoked by clients. By contradiction, assume that some correct replica logged a request not invoked by any client and let $r_{j}$ be the first correct replica to log such a request req in Step C3 of Chain. In case $j<f+1, r_{j} \operatorname{logs} r e q^{\prime}$ only if $r_{j}$ receives a CHAIN message with a MAC from the client, that is, only if some client invoked req, or if req is contained in some valid init history. On the other hand, if $j>f+1$, Lemma A.2 yields a contradiction with our assumption that $r_{j}$ is the first correct replica to log $r e q^{\prime}$.

Moreover, by Step C3, no replica logs the same request twice (every replica maintains $\left.t_{j}[c]\right)$. Hence, no request appears twice in any local history of a correct process, and consequently, no request appears twice in any commit history. In the case of abort histories, no request appears twice by construction (see Step P3, Section 4.2.2).

Termination. Since Chain uses the same panicking/aborting mechanism as Quorum/ZLight, the proof of Termination for ZLight/Quorum (Section A.1) applies.

Commit Order. Assume, by contradiction, that there are two different committed request req (by benign client $c$ ) and $r e q^{\prime}$ (by benign client $c^{\prime}$ ) with different commit histories $h_{r e q}$ and $h_{r e q^{\prime}}$ such that neither is the prefix of the other. By Lemma A.3, there is correct replica $r_{j} \in \Sigma_{\text {last }}$ that logged and executed req and req ${ }^{\prime}$ such that the state of 
$L H_{j}$ upon executing these requests is $h_{r e q}$ and $h_{r e q^{\prime}}$, respectively-a contradiction with Lemma A.1 (recall that this lemma extends to Chain as well).

Abort Order. Assume, by contradiction, that there is committed request req (by some benign client) with commit history $h_{r e q_{C}}$ and aborted request $r e q_{A}$ (by some benign client) with abort history $h_{r e q_{A}}$, such that $h_{r e q_{C}}$ is not a prefix of $h_{r e q_{A}}$. By Lemma A.3 and the assumption of at most $f$ faulty replicas, all correct replicas (at least one) from $\Sigma_{\text {last }} \log$ and execute $r e q_{C}$ and their state upon executing $r e q_{C}$ is $h_{r e q_{C}}$. Let $r_{j} \in \Sigma_{\text {last }}$ be a correct replica with the highest index $j$ among all correct replicas in $\Sigma_{\text {last }}$. By Lemma A.2, all correct replicas log all the requests in $h_{r e q_{C}}$ at the same positions these requests have in $h_{r e q_{C}}$. In addition, a correct replica logs all the requests belonging to $h_{r e q_{C}}$ before sending any ABORT message in Step P2; indeed, before sending any ABORT message, a correct replica must stop further execution of requests. Therefore, for every local history $L H_{j}$ that a correct replica sends in an ABORT message, $h_{r e q_{C}}$ is a prefix of $L H_{j}$.

Finally, by Step P3, a client that aborts a request waits for $2 f+1$ ABORT messages including at least $f+1$ from correct replicas. By construction of abort histories (Step P3), every commit history, including $h_{r e q_{C}}$, is a prefix of every abort history, including $h_{r e q_{A}}$-a contradiction.

Init Order. The proof is identical to the proof of ZLight/Quorum Init Order.

Progress (sketch). Chain guarantees to commit clients' requests under the same conditions as ZLight, that is, if there are no replica/link failures and no Byzantine client failures. Assuming that the message processing at processes takes $\Delta_{p}$ and communication time is bounded by $\Delta_{c}$, it is sufficient that clients set the timer $T$ triggered in Step C1 to $(3 f+2) \Delta$, where $\Delta=\Delta_{p}+\Delta_{c}$. Then, Progress of Chain is very simple to show, along the lines of ZLight Progress (Section A.1).

\section{REFERENCES}

Michael Abd-El-Malek, Gregory R. Ganger, Garth R. Goodson, Michael K. Reiter, and Jay J. Wylie. 2005. Fault-scalable Byzantine fault-tolerant services. In Proceedings of the Symposium on Operating Systems Principles (SOSP'05). ACM.

Marcos K. Aguilera, Svend Frolund, Vassos Hadzilacos, Stephanie L. Horn, and Sam Toueg. 2007. Abortable and query-abortable objects and their efficient implementation. In Proceedings of the ACM Symposium on Principles of Distributed computing (PODC'07).

Yair Amir, Brian A. Coan, Jonathan Kirsch, and John Lane. 2011. Prime: Byzantine replication under attack. IEEE Trans. Dependable Sec. Comput. 8, 4 (2011), 564-577.

Hagit Attiya, Rachid Guerraoui, and Petr Kouznetsov. 2005. Computing with reads and writes in the absence of step contention. In Proceedings of the International Conference on Distributed Computing (DISC'05).

Ken Birman, Dahlia Malkhi, and Robbert Van Renesse. 2010. Virtually Synchronous Methodology for Dynamic Service Replication. Technical Report MSR-TR-2010-151.

Romain Boichat, Partha Dutta, Svend Frölund, and Rachid Guerraoui. 2003. Deconstructing Paxos. SIGACT News Distrib. Comput. 34, 1 (2003), 47-67. DOI :http://dx.doi.org/10.1145/637437.637447

Francisco V. Brasileiro, Fabíola Greve, Achour Mostéfaoui, and Michel Raynal. 2001. Consensus in one communication step. In Proceedings of the International Conference on Parallel Computing Technologies (PaCT'01).

Miguel Castro and Barbara Liskov. 2002. Practical Byzantine fault tolerance and proactive recovery. ACM Trans. Comput. Syst. 20, 4 (Nov. 2002), 398-461. DOI : http://dx.doi.org/10.1145/571637.571640

Miguel Castro, Rodrigo Rodrigues, and Barbara Liskov. 2003. BASE: Using abstraction to improve fault tolerance. ACM Trans. Comput. Syst. 21, 3 (Aug. 2003), 236-269. DOI: http://dx.doi. org/10.1145/859716.859718

Tushar D. Chandra, Robert Griesemer, and Joshua Redstone. 2007. Paxos made live: An engineering perspective. In Proceedings of the ACM Symposium on Principles of Distributed Computing (PODC'07). ACM. DOI : http://dx.doi.org/10.1145/1281100.1281103

Wei Chen. 2007. Abortable Consensus and Its Application to Probabilistic Atomic Broadcast. Technical Report MSR-TR-2006-135. 
Allen Clement, Edmund Wong, Lorenzo Alvisi, Mike Dahlin, and Mirco Marchetti. 2009. Making Byzantine fault tolerant systems tolerate Byzantine faults. In Proceedings of the Symposium on Networked Systems Design and Implementation (NSDI'09).

James Cowling, Daniel Myers, Barbara Liskov, Rodrigo Rodrigues, and Liuba Shrira. 2006. HQ replication: A hybrid quorum protocol for Byzantine fault tolerance. In Proceedings of the Symposium on Operating Systems Design and Implementation (OSDI'06). USENIX Association. http://portal.acm.org/citation.cfm?id=1298455.1298473.

Dan Dobre and Neeraj Suri. 2006. One-step consensus with zero-degradation. In Proceedings of the 2004 International Conference on Dependable Systems and Networks (DSN'06).

Cynthia Dwork, Nancy Lynch, and Larry Stockmeyer. 1988. Consensus in the presence of partial synchrony. J. ACM 35, 2 (April 1988), 36. DOI : http://dx.doi.org/10.1145/42282.42283

Michael J. Fischer, Nancy A. Lynch, and Michael S. Paterson. 1985. Impossibility of distributed consensus with one faulty process. J. ACM 32, 2 (April 1985), 374-382.

Miguel Garcia, Alysson Bessani, Ilir Gashi, Nuno Neves, and Rafael Obelheiro. 2011. OS diversity for intrusion tolerance: Myth or reality? In Proceedings of the 2011 IEEE/IFIP 41st International Conference on Dependable Systems\&Networks (DSN'11). IEEE Computer Society, Washington, DC, 383-394. DOI : http://dx.doi.org/10.1109/DSN.2011.5958251

Ilir Gashi, Peter T. Popov, and Lorenzo Strigini. 2007. Fault tolerance via diversity for off-the-shelf products: A study with SQL database servers. IEEE Trans. Dependable Sec. Comput. 4, 4 (2007), 280-294.

Jim Gray. 1978. Notes on data base operating systems. In Operating Systems-An Advanced Course. Springer-Verlag, 393-481. http://dl.acm.org/citation.cfm?id=647433.723863

Rachid Guerraoui, Nikola Knežević, Vivien Quéma, and Marko Vukolić. 2008. The Next 700 BFT Protocols. Technical Report LPD-REPORT-2008-008. EPFL.

Rachid Guerraoui, Nikola Knežević, Vivien Quéma, and Marko Vukolić. 2010. The next 700 BFT protocols. In Proceedings of the ACM European Conference on Computer systems (EuroSys'10).

James Hendricks, Gregory R. Ganger, and Michael K. Reiter. 2007. Low-overhead byzantine fault-tolerant storage. In Proceedings of the Symposium on Operating Systems Principles (SOSP'07). ACM.

Maurice Herlihy and Jeannette M. Wing. 1990. Linearizability: A correctness condition for concurrent objects. ACM Trans. Program. Lang. Syst. 12, 3 (1990), 463-492.

Prasad Jayanti. 2003. Adaptive and efficient abortable mutual exclusion. In Proceedings of the ACM Symposium on Principles of Distributed Computing (PODC'03).

Rüdiger Kapitza, Johannes Behl, Christian Cachin, Tobias Distler, Simon Kuhnle, Seyed Vahid Mohammadi, Wolfgang Schröder-Preikschat, and Klaus Stengel. 2012. CheapBFT: Resource-efficient Byzantine fault tolerance. In Proceedings of the 7th ACM European Conference on Computer Systems (EuroSys'12). ACM, New York, NY, 295-308. DOI : http://dx.doi.org/10.1145/2168836.2168866

Ramakrishna Kotla, Lorenzo Alvisi, Mike Dahlin, Allen Clement, and Edmund Wong. 2010. Zyzzyva: Speculative Byzantine fault tolerance. ACM Trans. Comput. Syst. 27, 4, Article 7 (Jan. 2010), 39 pages. DOI : http://dx.doi.org/10.1145/1658357.1658358

Leslie Lamport. 2003. Lower bounds for asynchronous consensus. In Proceedings of the International Workshop on Future Directions in Distributed Computing (FuDiCo'03).

Leslie Lamport. 2009. The PlusCal algorithm language. In Proceedings of the 6th International Colloquium on Theoretical Aspects of Computing (ICTAC). 36-60.

Leslie Lamport, Dahlia Malkhi, and Lidong Zhou. 2010. Reconfiguring a state machine. SIGACT News 41, 1 (2010), 63-73.

Fernando Pedone. 2001. Boosting system performance with optimistic distributed protocols. Comput. J. 34, 12 (2001), 80-86. DOI : http://dx.doi.org/10.1109/2.970581

Fred B. Schneider. 1990. Implementing fault-tolerant services using the state machine approach: A tutorial. ACM Comput. Surv. 22, 4 (Dec. 1990), 299-319. DOI : http://dx.doi.org/10.1145/98163.98167

Bianca Schroeder, Adam Wierman, and Mor Harchol-Balter. 2006. Open versus closed: A cautionary tale. In Proceedings of the Symposium on Networked Systems Design and Implementation (NSDI). 18-18.

Atul Singh, Tathagata Das, Petros Maniatis, Peter Druschel, and Timothy Roscoe. 2008. BFT protocols under fire. In Proceedings of the Symposium on Networked Systems Design and Implementation (NSDI'08). USENIX Association.

Sam Toueg. 1984. Randomized Byzantine agreements. In Proceedings of the 3rd Annual ACM Symposium on Principles of Distributed Computing. 163-178.

Robbert van Renesse and Rachid Guerraoui. 2010. Replication techniques for availability. In Replication, B. Charron-Bost, F. Pedone, and A. Schiper (Eds.). Springer-Verlag, 19-40. http://dl.acm.org/citation. cfm?id=2172338.2172340 
Robbert van Renesse and Fred B. Schneider. 2004. Chain replication for supporting high throughput and availability. In Proceedings of the Symposium on Operating Systems Design and Implementation (OSDI'04).

Giuliana Santos Veronese, Miguel Correia, Alysson Neves Bessani, and Lau Cheuk Lung. 2009. Spin one's wheels? Byzantine fault tolerance with a spinning primary. In Proceedings of International Symposium on Reliable Distributed Systems (SRDS'09). IEEE Computer Society. DOI: http://dx.doi. org/10.1109/SRDS.2009.36

Giuliana Santos Veronese, Miguel Correia, Alysson Neves Bessani, Lau Cheuk Lung, and Paulo Veríssimo. 2013. Efficient byzantine fault-tolerance. IEEE Trans. Comput. 62, 1 (2013), 16-30.

Received May 2012; revised February 2014; accepted July 2014 